\title{
KINEMATIC GEOMETRY; AN AXIOMATIG SYSTEM FOR MINKOWSKI SPACE-TIME
}

\author{
M. L. URQUHART IN MEMORIAM
}

G. SZEKERES

(Received 1 August 1966, revised 7 January 1967)

\section{Introduction}

The aim of this paper is, briefly, an axiomatization of relativistic kinematics. Before stating the aims in more precise terms, a few words about the origins of the paper will be necessary. The idea of a revision of the axiomatic foundations of relativistic kinematics came up in discussions with the late M. L. Urquhart at the 1963 (3rd) Summer Research Institute of the Australian Mathematical Society, and it was a suggestion by Urquhart which started off the present investigation. Following his suggestion I prepared a preliminary draft containing the outlines of an axiomatic system for Minkowski space-time and passed it on to him. Shortly before his death Urquhart asked Professor D. Elliott to send the manuscript back to me and it was this manuscript which formed the nucleus of the present paper.

It would be rather difficult to pinpoint Urquhart's actual contribution to the paper as the material and the form of presentation have changed considerably since these preliminary discussions; there is however one particular result, Theorem 14, which is entirely his. It tells us that if the observer $A$ has relative speed $v$ with respect to observer $B$ then $B$ has the same speed $v$ with respect to $A$. I owe to Urquhart the observation that this is not a hypothesis but it can be deduced from more primitive assumptions. The proof of Theorem $\mathbf{1 4}$ is modelled after his original reasoning.

The deductive structure of relativistic kinematics (or its geometrical model, Minkowski space -time) has of course been examined before. The traditional approach, initiated by Hermann Weyl [2] and followed also in a recent axiomatization by Walter Noll [1], is to regard Minkowski spacetime as an inner product space with appropriate signature, essentially an extension of the Euclidean system by "Minkowskian chronometry" (Noll's terminology).

From the epistemological point of view this method of approach leaves 
much to be desired. In physical space-time, 3 -space is essentially a space of simultaneity relative to a fixed observer and its properties can only be inferred indirectly, from kinematic observations relating to observers and light signals. It seems therefore inappropriate to build up kinematics from axioms which make use of assumed properties of 3-space, either explicitly or under the disguise of an inner product space.

An example which goes back to Gauss will illustrate the point. If the angles of a huge triangle formed for example by three fixed stars were measured by direct visual observation then according to special relativity they will add up to two right angles. What is the source of this assertion? The nature of large-scale optical measurements is vastly different from the kind of empirical evidence upon which Euclidean geometry was founded and their outcome can hardly be regarded as "self-evident truth" in the sense of the ancient Greek geometers, unless one is prepared to accept the (long discredited) Kantian dogma at its face value that Euclidean geometry is the only possible form of space perception. In a non-Kantian approach to relativistic kinematics the Euclidean space structure must appear as a consequence of the kinematical axioms rather than an a priori structure built into the system.

After this preamble let us state our aim more definitely. It is to set up a system of axioms for Minkowski space-time which refer solely to kinematic notions such as time and motion, but which make no assumptions about 3-space itself.

The natural primary objects of such a "kinematic" geometry are events, world lines and time. World lines are classified into time-like and light-like; the former represent inertial observers or free particles, the latter undeflected light pulses. For convenience we shall call monochromatic light pulses photons, to indicate the existence of a natural "clock" which photons carry along by virtue of their energy or frequency. Similarly we assume that particles are endowed with a time-keeping device. Space-like world lines will not appear in the system as they have no immediate connection with kinematic experience.

Each axiom will be accompanied by a descriptive name and by a brief indication of the kinematical phenomenon with which it is associated. The axioms will be formulated in several stages and at each stage the consequences developed as fully as possible so as to highlight the deductive structure of the theory.

Questions of consistency and redundancy will not be considered. Consistency of the axioms is almost trivial from the fact that they are valid for suitable entities in a pseudo-Euclidean geometry with signature $3+1$. Independence is a more subtle question and it is quite possible that some of the axioms could be replaced by weaker ones or even discarded. 
The real number line has been adopted throughout as the standard model for time-ordering of events on world lines. This is merely for convenience and for greater flexibility; as long as we are not dealing with dynamics and the necessity for analysis does not arise, any dense subfield of the reals such as the rational field could equally well be employed for the construction of a geometrical model of space-time.

The present treatment has a certain affinity with A. A. Robb's axiomatic system for space and time [3], and it is necessary to say a few words about the relative position of the two systems. Robb's guiding principle is mathematical economy; in this he succeeds admirably. All postulates are stated in terms of a single relation (before-after) between the elements of his set, and the whole structure of Minkowski space-time is derived from the properties of this one relation. The justification of his postulates is primarily on mathematical grounds and not in their relation to direct kinematical experience which is often quite remote. The following example is characteristic of the difference in the attitudes of the two treatments. Theorem 3 of the present paper on the arrow of time, which is the outcome of a long line of arguments using the first six axioms on configurations of world lines, appears as Postulate 3 at the very beginning of Robb's system. On the other hand world lines (the most direct objects of kinematical experience) are derived quantities in Robb's system and they do not appear until Postulate 9 ("optical lines") and Postulate 14 ("inertia lines"). Moreover, some of his postulates refer to space-like pairs of events and space-like world lines, both of which have been completely avoided in the formulation of the present axioms.

\section{Axioms of incidence and inertia}

Minkowski space-time shall consist of the following:

(i) A space $\mathfrak{W}$ whose are called events. Thus $p \in \mathfrak{W}$ means: $p$ is an event.

(ii) A set $\mathscr{N}$ of one to one mappings

$$
N: \mathfrak{T} \rightarrow \mathfrak{W}
$$

from the real line

$$
\mathfrak{I}=\{\boldsymbol{\tau} ;-\infty<\tau<\infty\}
$$

into $\mathfrak{B}$. Elements of $\mathscr{N}$ are called world lines and $\tau$ is called local time on $N$. Thus

$$
N \in \mathscr{N}, \tau \in \mathfrak{I} \Rightarrow p=N(\tau) \in \mathfrak{W},
$$

and $\tau_{1} \neq \tau_{2} \Rightarrow N\left(\tau_{1}\right) \neq N\left(\tau_{2}\right)$. The set of events $\{N(\tau) ;-\infty<\tau<\infty\}$ is denoted by $|N|$. If $p \in|\boldsymbol{N}|$, we say that the event $p$ lies on the world line $N$, or that $N$ goes through $p$. 
(iii) Two subsets $\mathcal{O}$ and $\mathscr{P}$ of $\mathscr{N}$ such that

$$
\mathcal{O} \cap \mathscr{P} \neq \emptyset, \mathcal{O} \cup \mathscr{P}=\mathscr{N} \text {. }
$$

Mappings $\boldsymbol{O} \in \mathcal{O}$ are called particles, mappings $\boldsymbol{P} \in \mathscr{P}$ are called photons. Thus each $N \in \mathscr{N}$ is either a particle or a photon, but not both.

(iv) A subset $\mathscr{N}^{*}$ of $\mathscr{N}$ whose members are called standard world lines; elements of $\mathcal{O}^{*}=\mathcal{O} \cap \mathscr{N}^{*}$ are called standard particles, elements of $\mathscr{P}^{*}=\mathscr{P} \cap \mathscr{N}^{*}$ standard photons.

In the present section only the first three entities will be considered. A substantial portion of space-time relations can be stated without taking into account the evenness of time flow but merely assuming a time ordering of events on the world lines. Standard (uniform) local time will only be introduced in the next section when it comes to the discussion of relative speeds.

\section{Al (AXIOM OF EXISTENCE)}

The set $\mathcal{O}$ is not empty. Moreover to every $\boldsymbol{O} \in \mathcal{O}$ there is a $q \in \mathfrak{M}$ such that $q \notin|\boldsymbol{O}|$.

The axiom states that there is at least one particle and an event through which it does not pass. In particular $\mathfrak{W}$ is not empty and by (ii) there are infinitely many distinct events.

\section{A2 (AXIOM OF TIME ORDERING)}

Let $\phi: \mathfrak{T} \rightarrow \mathfrak{I}$ be a sense-preserving homeomorphism of $\mathfrak{I}$ onto $\mathfrak{T}$, i.e. $\phi(\tau)$ a continuous striculy increasing real valued function of $\tau$ with

$$
\lim _{\tau \rightarrow-\infty} \phi(\tau)=-\infty, \lim _{\tau \rightarrow \infty} \phi(\tau)=\infty .
$$

Then $\boldsymbol{O} \in \mathcal{O} \Rightarrow \boldsymbol{O}^{\prime}=\boldsymbol{O} \circ \phi \in \mathcal{O}, \boldsymbol{P} \in \mathscr{P} \Rightarrow \boldsymbol{P}^{\prime}=\boldsymbol{P} \circ \phi \in \mathscr{P}$.

We say that $\boldsymbol{O}^{\prime}$ is homographic to $\boldsymbol{O}, \boldsymbol{P}^{\prime}$ is homographic to $\boldsymbol{P}$. Clearly homography is an equivalence relation; a class of homographic particles is called an observer and a class of homographic photons a light signal (l.s.). We denote by $\{\boldsymbol{O}\}$ the observer represented by the particle $\boldsymbol{O}$, and by $\{\boldsymbol{P}\}$ the l.s. represented by the photon $\boldsymbol{P}$.

Homographic world lines pass through exactly the same events (i.e. $|\boldsymbol{N}|=|\boldsymbol{N} \circ \phi|)$ and differ only in their local times. Axiom A2 establishes a time-ordering of events on an observer or 1.s. independently of the representing particle or photon, i.e. of the local time used. It also establishes a real-line topology on world lines, it is in fact consistent with the assumption that $N: \mathfrak{T} \rightarrow|N|$ is a homeomorphism.

\section{A3 (First AXIOM OF INCIDENCE)}

Two non-homographic world lines intersect at most at one event. 
Hence they either do not intersect at all, or they have exactly one event in common. It follows from the axiom that given two distinct events $p_{1}$ and $p_{2}$, there either passes exactly one class of homographic world lines through them, or there is no world line joining $p_{1}$ and $p_{2}$. In the first case we call $\left(p_{1}, p_{2}\right)$ an ordered pair and write $p_{1}<p_{2}$ if $p_{1}=N\left(\tau_{1}\right), p_{2}=N\left(\tau_{2}\right)$, $\tau_{1}<\tau_{2}$, for some $N \in \mathscr{N}$. In particular the ordered pair $\left(p_{1}, p_{2}\right)$ is called time-like iff there is an observer $\{\boldsymbol{O}\}$ such that $p_{\mathbf{1}} \in|\boldsymbol{O}|, p_{\mathbf{2}} \in|\boldsymbol{O}|$, and lightlike iff. there is a l.s. $\{\boldsymbol{P}\}$ such that $p_{1} \in|\boldsymbol{P}|, p_{\mathbf{2}} \in|\boldsymbol{P}|$. A non-ordered pair of events is called space-like. Axioms A2 and A3 show that these three possibilities are mutually exclusive. Absence of a world line joining $p_{1}$ and $p_{2}$ indicates that in the traditional treatment there passes a space-like world line through these events. We call the distinct events $p_{1}, p_{2}, p_{3}$ collinear iff there is a world line $\boldsymbol{N}$ passing through them.

\section{A4 (AxIOM of CONNECTEDNESS)}

Given an observer $\{\boldsymbol{O}\}$ and an event $q \notin|\boldsymbol{O}|$, there are exactly two light signals $\left\{\boldsymbol{P}_{1}\right\},\left\{\boldsymbol{P}_{2}\right\}$ through $q$ and intersecting $\boldsymbol{O}$ in the distinct events $p_{1}, p_{2}$. Furthermore if $p_{1}<p_{2}$ then $p_{1}<q, q<p_{2}$.

The axiom states that an observer receives a light signal from every event of the Universe and can also send a light signal so as to reach any given event. Topologically it expresses connectedness of space-time. A4 is the decisive axiom which distinguishes Einsteinean from Galilean relativity; it is clearly invalid in a Universe in which light travels at infinite speed or in which an observer may move faster than light.

\section{A5 (SECOND AXIOM of INCIDENCE)}

If the distinct light signals $\left\{\boldsymbol{P}_{\mathbf{1}}\right\},\left\{\boldsymbol{P}_{\mathbf{2}}\right\}$ intersect at $q$, and $p_{\mathbf{1}} \in\left|\boldsymbol{P}_{\mathbf{1}}\right|, \boldsymbol{p}_{\mathbf{2}} \in\left|\boldsymbol{P}_{\mathbf{2}}\right|$ such that $p_{1}<q, q<p_{2}$, then there is an observer $\{\boldsymbol{O}\}$ such that $p_{1} \in|\boldsymbol{O}|$, $p_{2} \in|\boldsymbol{O}|$.

This in a sense is a converse of Axiom A4. $\{\boldsymbol{O}\}$ is uniquely determined, according to A3. In physical terms the axiom states that if a light pulse is reflected on a mirror then any point on the path of the reflected pulse can be reached from any point on the path of the incident pulse by a suitably moving observer. Two intersecting light signals are said to be conjugate.

\section{A6 (Third AXIOM of InCIDENCE)}

Given an observer $\{\boldsymbol{O}\}$, a light signal $\{\boldsymbol{P}\}$ intersecting $\boldsymbol{O}$, and an event $q$ not on $\boldsymbol{O}$ but on $\boldsymbol{P}$, there is a light signal $\left\{\boldsymbol{P}^{\prime}\right\}$ which passes through $q$ and intersects $\boldsymbol{P}$.

This light signal is uniquely determined; for let $\left\{\boldsymbol{P}_{1}\right\}$ be a second light signal through $q$, intersecting $\boldsymbol{P}$ in $p_{1}>p$. Then if $q<p$, there goes an 
observer from $q$ to $p_{1}$, if $q>p_{1}$ there goes an observer from $p$ to $q$, and if $q>p, q<p_{1}$ then there goes an observer from $p$ to $p_{1}$, by A4, contrary to A3.

Axiom A6 splits events not on $\boldsymbol{P}$ into three disjoint classes: those for which $p=|\boldsymbol{P}| \cap\left|\boldsymbol{P}^{\prime}\right|<q$, those for which $q<p$, and those for which $\boldsymbol{P}^{\prime}$ does not exist. Thus there is a clear separation into past, future and "simultaneity" of world events relative to a light signal. In the first two cases we say that $q$ is ordered with respect to $\boldsymbol{P}$ (is before or after $\boldsymbol{P}$ ).

Theorem 1. Let $p_{1}, p_{2}, p_{3}$ be distinct events not on the same world line such that all three pairs $\left(p_{i}, p_{j}\right), 1 \leqq i<j \leqq 3$ are ordered; then with $a$ suitable numbering of the events $p_{i}$ we have

$$
p_{1}<p_{2}, p_{2}<p_{3}, p_{1}<p_{3},
$$

and one of the following is true:

1. $\left(p_{1}, p_{3}\right)$ is time-like, $\left(p_{1}, p_{2}\right)$ and $\left(p_{2}, p_{3}\right)$ are light-like.

2a. $\left(p_{1}, p_{3}\right),\left(p_{2}, p_{3}\right)$ are time-like, $\left(p_{1}, p_{2}\right)$ light-like.

2b. $\left(p_{1}, p_{2}\right),\left(p_{1}, p_{3}\right)$ are time-like, $\left(p_{2}, p_{3}\right)$ light-like.

3. $\left(p_{1}, p_{2}\right),\left(p_{2}, p_{3}\right),\left(p_{1}, p_{3}\right)$ are time-like.

CoRollary. If $p_{1}, p_{2}, p_{3}$ are not collinear, $\left(p_{1}, p_{2}\right)$ and $\left(p_{2}, p_{3}\right)$ are lightlike, and $p_{1}<p_{2}, p_{3}<p_{2}$ or $p_{2}<p_{1}, p_{2}<p_{3}$, then $\left(p_{1}, p_{3}\right)$ is space-like.

Proof. It follows immediately from axiom $\mathrm{A} 6$ that at least one of the pais $\left(p_{i}, p_{j}\right)$ is time-like. Axiom A4 supplies an example for case 1 and rules out all other possibilities in which two of the pairs $\left(p_{i}, p_{j}\right)$ is lightlike and one is time-like.

Now suppose that $\left(p_{1}, p_{2}\right)$ and $\left(p_{2}, p_{3}\right)$ are time-like, $\left(p_{1}, p_{3}\right)$ is lightlike, and $p_{1}<p_{3}$. Let $\boldsymbol{O}_{1} \in \mathcal{O}$ be through $\left(p_{1}, p_{2}\right), \boldsymbol{O}_{2} \in \mathcal{O}$ be through $\left(p_{2}, p_{3}\right), \boldsymbol{P} \in \mathscr{P}$ through $\left(p_{1}, p_{3}\right)$. By A4 there exists an event $q$ on $\boldsymbol{P}$ and $\boldsymbol{P}^{\prime} \in \mathscr{P}$ such that $\boldsymbol{P}^{\prime}$ joins $p_{\mathbf{2}}$ and $q$. Suppose first that $q$ is between $p_{1}$ and $p_{3}$. Then if $q<p_{2}$, the configuration $\boldsymbol{O}_{2}, q$ conflicts with $\mathrm{A4}$, and if $p_{2}<q$ then $O_{1}, q$ conflicts with A4. We may suppose therefore that either $p_{1}<p_{3}<q$ or $q<p_{1}<p_{3}$. In the first case we have, by A4, $q<p_{2}$, $p_{3}<p_{2}, p_{1}<p_{2}$, hence case 2a (with $p_{2}, p_{3}$ interchanged). In the second case we have similarly $p_{2}<q, p_{2}<p_{1}, p_{2}<p_{3}$, which is case $2 \mathrm{~b}$ (with $p_{1}, p_{2}$ interchanged). This settles all cases with two time-like pairs and one light-like pair.

Finally consider the case when all pairs $\left(p_{i}, p_{j}\right)$ are time-like. Let $\boldsymbol{O} \in \mathcal{O}$ be through $\left(p_{2}, p_{3}\right)$ and suppose that $p_{1}<p_{2}, p_{2}<p_{3}, p_{3}<p_{1}$. Let $q \in|\boldsymbol{O}|$ be such that $\left(p_{1}, q\right)$ is light-like and $p_{1}<q$. If $q<p_{3}$ then the triangle $\left(p, q_{3}, p_{1}\right)$ is prohibited, and if $p_{3}<q$ then $\left(p_{1}, p_{2}, q\right)$ is prohibited by the previous result. Hence case 3 is the only remaining possibility. 
THeOREM 2. Let $p_{1}, p_{2}, p_{3}$ be non-collinear events such that $\left(p_{1}, p_{2}\right)$ and $\left(p_{2}, p_{3}\right)$ are ordered and $p_{1}<p_{2}, p_{2}<p_{3}$. Then $\left(p_{1}, p_{3}\right)$ is time-like and $p_{1}<p_{3}$.

Proof. If both $\left(p_{1}, p_{2}\right)$ and $\left(p_{2}, p_{3}\right)$ are light-like then the statement follows from A5. If $\left(p_{1}, p_{2}\right)$ is light-like, $\left(p_{2}, p_{3}\right)$ time-like, then $\left(p_{1}, p_{3}\right)$ is not light-like since this would conflict with Theorem 1. Let $\{\boldsymbol{P}\}$ be the 1.s. through $\left(p_{1}, p_{2}\right)$ and $\left\{\boldsymbol{P}^{\prime}\right\}$ the l.s. through $p_{3}$, intersecting $\boldsymbol{P}$ at $q$. Then $p_{2}<q, q<p_{3}$, for otherwise the triangle $\left(q, p_{2}, p_{3}\right)$ would conflict with Theorem 1. But $\left(p_{1}, q\right),\left(q, p_{3}\right)$ represents the previous case.

If $\left(p_{1}, p_{2}\right)$ is time-like, $\left(p_{2}, p_{3}\right)$ light-like, and $\{\boldsymbol{P}\}$ a l.s. through $\left(p_{2}, p_{3}\right)$, $\left\{\boldsymbol{P}^{\prime}\right\}$ a l.s. through $p_{1}$ and intersecting $\boldsymbol{P}$ in $q$, we find by a similar argument that the only possibility is $p_{1}<q, q<p_{3}$, and we have again the first case.

Finally if both $\left(p_{1}, p_{2}\right)$ and $\left(p_{2}, p_{3}\right)$ are time-like and $\{\boldsymbol{O}\}$ an observer through $\left(p_{2}, p_{3}\right)$ then take the l.s. from $p_{1}$ to $\boldsymbol{O}$, intersecting $\boldsymbol{O}$ at $q$. By Theorem 1 we must have $p_{1}<q, q<p_{3}$ which is the second case treated previously.

The theorem shows that the relation $p<q$ is a partial ordering of $\mathfrak{W}$.

TheOREM 3. (Arrow of time). Let $p_{1}, p_{2}, \cdots, p_{k}(k>1)$ be a sequence of events such that $\left(p_{i}, p_{i+1}\right)$ is ordered, $p_{i}<p_{i+1}$ for $i=1, \cdots, k-1$. Then $\left(p_{1}, p_{k}\right)$ is ordered and $p_{1}<p_{k}$.

Proof. If the events $p_{i}$ are collinear then the statement is trivial (from A2 and A3). Otherwise the theorem is obtained by a repeated application of Theorem 2 .

Now let $\{\boldsymbol{O}\}$ be an observer, $\{\boldsymbol{P}\}$ a l.s. intersecting $\boldsymbol{O}$ in $q$; let $\boldsymbol{p}_{\mathbf{1}}, \boldsymbol{p}_{\mathbf{2}}$ be events on $\boldsymbol{P}$, distinct from $q$ but not necessarily from each other. By A4 there exist unique light signals $\left\{\boldsymbol{P}_{\mathbf{1}}\right\},\left\{\boldsymbol{P}_{\mathbf{2}}\right\}$ through $p_{\mathbf{1}}, p_{\mathbf{2}}$, conjugate to $\{\boldsymbol{P}\}$, which intersect $\boldsymbol{O}$. We denote the configuration so obtained by $[\boldsymbol{O}, \boldsymbol{P}$; $\left.\boldsymbol{P}_{\mathbf{1}}, \boldsymbol{P}_{\mathbf{2}}\right]$. From Theorem 1 it follows that $\left\{\boldsymbol{P}_{\mathbf{1}}\right\}$ and $\left\{\boldsymbol{P}_{\mathbf{2}}\right\}$ are either identical or they do not intersect; they are said to be parallel light signals and denoted $\left\{\boldsymbol{P}_{\mathbf{1}}\right\} \mid\left\{\boldsymbol{P}_{2}\right\}$. Thus parallelity of $\left\{\boldsymbol{P}_{\mathbf{1}}\right\},\left\{\boldsymbol{P}_{\mathbf{2}}\right\}$ signifies that there exists some configuration $\left[\boldsymbol{O}, \boldsymbol{P} ; \boldsymbol{P}_{\mathbf{1}}, \boldsymbol{P}_{\mathbf{2}}\right]$ of which $\boldsymbol{P}_{\mathbf{1}}, \boldsymbol{P}_{\mathbf{2}}$ are the last two members. Clearly $\left\{\boldsymbol{P}_{\mathbf{1}}\right\} \mid\left\{\boldsymbol{P}_{\mathbf{1}}\right\}$ for all $\boldsymbol{P}_{\mathbf{1}} \in \mathscr{P}$ and $\left\{\boldsymbol{P}_{\mathbf{1}}\right\} \mid\left\{\boldsymbol{P}_{\mathbf{2}}\right\}$ implies $\left\{\boldsymbol{P}_{\mathbf{2}}\right\} \mid\left\{\boldsymbol{P}_{\mathbf{1}}\right\}$. We shall find later (from other axioms) that parallelity is an equivalence relation.

\section{A7 (AXIOM OF INERTIA)}

Given a light signal $\left\{\boldsymbol{P}_{\mathbf{1}}\right\}$ and an event $q$ ordered with respect to $\boldsymbol{P}_{\mathbf{1}}$, there is exactly one light signal $\left\{\boldsymbol{P}_{\mathbf{2}}\right\}$ through $q$ which is parallel to $\left\{\boldsymbol{P}_{\mathbf{1}}\right\}$.

Although the axiom has the form of an axiom of parallelity for photons, it expresses the first half of the law of inertia, namely that particles and 
photons move on a straight path without torsion. The second half of the law of inertia, namely that they move with an even speed, will be expressed later.

The following two theorems bring out the law of inertia more explicitly.

Theorem 4. (Law of inertia for photons). Let $\left\{\boldsymbol{P}_{\mathbf{1}}\right\},\left\{\boldsymbol{P}_{\mathbf{2}}\right\}$ be distinct parallel light signals derived from the configuration $\left[\boldsymbol{O}, \boldsymbol{P} ; \boldsymbol{P}_{\mathbf{1}}, \boldsymbol{P}_{\mathbf{2}}\right]$. Let $q \in\left|\boldsymbol{P}_{1}\right|, q \notin|\boldsymbol{O}|$, and $\left\{\boldsymbol{P}^{\prime}\right\}$ the unique l.s., conjugate to $\left\{\boldsymbol{P}_{1}\right\}$, which by $A \mathbf{4}$ passes through $q$ and intersects $\boldsymbol{O}$. Then $\left\{\boldsymbol{P}^{\prime}\right\}$ also intersects $\boldsymbol{P}_{\mathbf{2}}$.

Proof. Let $q^{\prime}=|\boldsymbol{O}| \cap\left|\boldsymbol{P}^{\prime}\right|$, and $\left\{\boldsymbol{P}^{\prime \prime}\right\}$ the unique l.s. through $q^{\prime}$ which by A6 intersects $\boldsymbol{P}_{\mathbf{2}}$. Then both $\left\{\boldsymbol{P}^{\prime}\right\}$ and $\left\{\boldsymbol{P}^{\prime \prime}\right\}$ pass through $q^{\prime}$ and are parallel to $\{\boldsymbol{P}\}$, hence by A7 they are identical, and so $\left\{\boldsymbol{P}^{\prime}\right\}$ intersects $\boldsymbol{P}_{\mathbf{2}}$.

Theorem 5. (First law of inertia of particles). Let $\left\{\boldsymbol{P}_{\mathbf{1}}\right\},\left\{\boldsymbol{P}_{\mathbf{2}}\right\}$ be distinct parallel light signals derived from the configuration $\left[\boldsymbol{O}, \boldsymbol{P} ; \boldsymbol{P}_{\mathbf{1}}, \boldsymbol{P}_{\mathbf{2}}\right]$, and $\left\{\boldsymbol{O}^{\prime}\right\}$ an observer which intersects $\boldsymbol{P}$ and $\boldsymbol{P}_{\mathbf{1}}$ in the distinct events $p$ and $p_{\mathbf{1}}$. Then $\left\{\boldsymbol{O}^{\prime}\right\}$ also intersects $\boldsymbol{P}_{2}$.

Proof. Let $p_{2}=|\boldsymbol{P}| \cap\left|\boldsymbol{P}_{2}\right|$ and $\left\{\boldsymbol{P}_{2}^{\prime}\right\}$ the unique 1.s., conjugate to $\boldsymbol{P}$, which by A4 passes through $p_{\mathbf{2}}$ and intersects $\boldsymbol{O}^{\prime}$. Then $\left\{\boldsymbol{P}_{\mathbf{2}}^{\prime}\right\} \mid\left\{\boldsymbol{P}_{\mathbf{1}}\right\}$, because of the configuration $\left[\boldsymbol{O}^{\prime}, \boldsymbol{P} ; \boldsymbol{P}_{\mathbf{1}}, \boldsymbol{P}_{\mathbf{2}}^{\prime}\right]$, and both $\left\{\boldsymbol{P}_{\mathbf{2}}\right\}$ and $\left\{\boldsymbol{P}_{\mathbf{2}}^{\prime}\right\}$ pass through $p_{2}$, hence by A7 they are identical. Consequently $\left\{\boldsymbol{P}_{2}\right\}$ intersects $\boldsymbol{O}^{\prime}$.

We denote by $\mathfrak{B}(\boldsymbol{O}, \boldsymbol{P})$ the union of all light signals $\left\{\boldsymbol{P}^{\prime}\right\}, \boldsymbol{P}^{\prime} \in \mathscr{P}$, where $\boldsymbol{P}^{\prime}$ intersects $\boldsymbol{O}$ and $\left\{\boldsymbol{P}^{\prime}\right\} \mid\{\boldsymbol{P}\}$, and by $|\mathfrak{B}(\boldsymbol{O}, \boldsymbol{P})|$ the union of all sets of events $\left|\boldsymbol{P}^{\prime}\right|$ where $\left\{\boldsymbol{P}^{\prime}\right\} \in \mathfrak{B}(\boldsymbol{O}, \boldsymbol{P})$. By A7 there passes exactly one $\left\{\boldsymbol{P}^{\prime}\right\} \in \mathfrak{B}(\boldsymbol{O}, \boldsymbol{P})$ through every $p \in|\boldsymbol{O}|$ and $\mathfrak{B}(\boldsymbol{O}, \boldsymbol{P})=\mathfrak{B}\left(\boldsymbol{O}, \boldsymbol{P}^{\prime}\right)$ for every such $\boldsymbol{P}^{\prime}$. If $q \in\left|\boldsymbol{P}^{\prime}\right|$, $q \notin|\boldsymbol{O}|$, and $\left\{\boldsymbol{P}^{*}\right\}$ is the second l.s. through $q$ which intersects $\boldsymbol{O}$ then by Theorem 4, $\left|\boldsymbol{P}^{*}\right| \subset|\mathfrak{B}(\boldsymbol{O}, \boldsymbol{P})|$ and $\left|\mathfrak{B}\left(\boldsymbol{O}, \boldsymbol{P}^{*}\right)\right|=|\mathfrak{B}(\boldsymbol{O}, \boldsymbol{P})|$. We call $\mathfrak{B}^{*}=\mathfrak{B}\left(\boldsymbol{O}, \boldsymbol{P}^{*}\right)$ and $\mathfrak{B}=\mathfrak{B}(\boldsymbol{O}, \boldsymbol{P})$ conjugate pseudoplanes; their members go through exactly the same events. If $\left\{\boldsymbol{O}^{\prime}\right\}$ is an observer which has two events in common with $|\mathfrak{B}(\boldsymbol{O}, \boldsymbol{P})|$ then by Theorem $\mathbf{5}\left|\boldsymbol{O}^{\prime}\right| \subset|\mathfrak{B}(\boldsymbol{O}, \boldsymbol{P})|$ and $\mathfrak{B}\left(\boldsymbol{O}^{\prime}, \boldsymbol{P}\right)=\mathfrak{B}(\boldsymbol{O}, \boldsymbol{P})$. Hence $\left|\mathfrak{B}\left(\boldsymbol{O}^{\prime}, \boldsymbol{P}^{\prime}\right)\right|=|\mathfrak{B}(\boldsymbol{O}, \boldsymbol{P})|$ for any observer $\left\{\boldsymbol{O}^{\prime}\right\}$ and l.s. $\left\{\boldsymbol{P}^{\prime}\right\}$ which lie in $|\mathfrak{B}(\boldsymbol{O}, \boldsymbol{P})|$. We denote by $\mathscr{V}(\boldsymbol{O})$ the set of all pseudoplanes ${ }^{1} \mathfrak{B}(\boldsymbol{O}, \boldsymbol{P})$ where $\{\boldsymbol{P}\}$ is a l.s. intersecting $\boldsymbol{O}$.

\section{Standard time and synchronization}

The local time on world lines has so far only been restricted by the conditions of Axiom A2. Clearly with such an arbitrary time it is not possible to formulate significant statements about relative speeds and kinematic measurements. In order to take account of the evenness of time flow, we

1 Called inertia planes by Robb. 
introduce now the concept of standard local time (s.l.t.) on world lines.

On each class $\{\boldsymbol{N}\}$ of homographic world lines we distinguish a nonempty subset $\{\boldsymbol{N}\}^{*}$ of standard riorld lines $N^{*} \in \mathscr{N}^{*}$ with the following property:

A8 (AXIOM OF STANDARD TIME)

If $N \in \mathscr{N}$ and $N_{1}^{*}, N_{2}^{*} \in\{N\}^{*}$ then $N_{2}^{*}=N_{1}^{*} \circ A$ where $\Lambda: \mathfrak{I} \rightarrow \mathfrak{I}$ is the linear homeomorphism

$$
\Lambda(t)=\gamma t+\delta, \gamma>0, \delta \text { real. }
$$

Conversely, $N_{1}^{*} \in\{N\}^{*}$ implies $N_{1}^{*} \circ \Lambda \in\{N\}^{*}$ for all $\Lambda(t)$ of the form (2).

The axiom expresses the basic property of standard time, namely that two standard local times on a world line (observer or 1.s.) differ only in scale and time origin. In order to make the definition meaningful, it is necessary to relate the standard times on non-homographic world lines.

Let $q$ be an arbitrary event not on the standard particle $\boldsymbol{O}$, and $\{\boldsymbol{P}\},\left\{\boldsymbol{P}^{*}\right\}$ the two light signals through $q$ which, by A4, intersect $\boldsymbol{O}$. Let $\boldsymbol{O}\left(t_{1}\right)=|\boldsymbol{O}| \cap|\boldsymbol{P}|, \quad \boldsymbol{O}\left(t_{2}\right)=|\boldsymbol{O}| \cap\left|\boldsymbol{P}^{*}\right| ; \quad$ by A3, $t_{1} \neq t_{2}$. We define $t(\boldsymbol{O}, q)=\frac{\mathbf{1}}{2}\left(t_{1}+t_{2}\right)$ as the synchronized time of the event $q$ relative to $\boldsymbol{O}$, and $d(\boldsymbol{O}, q)=\frac{1}{2}\left|t_{1}-t_{2}\right|$ the synchronized distance of $q$ from $\boldsymbol{O}$. These definitions are in agreement with Einstein's definition of time and distance; the speed of light is taken to be unity.

\section{A9 (AXIOM OF SYNCHRONIZATION)}

Let $\boldsymbol{O} \in \mathcal{O}^{*}$ and $\{\boldsymbol{P}\}$ any light signal. Then there is a unique standard photon $\boldsymbol{P}^{*} \in\{\boldsymbol{P}\}^{*}$ such that

$$
t\left(O, \boldsymbol{P}^{*}(\tau)\right)=\tau \text { for every } \tau \in \mathfrak{I} .
$$

The axiom asserts two things: first, that the synchronized time of the events on $\{\boldsymbol{P}\}$ (relative to a standard particle $\boldsymbol{O}$ ) is an admissible local time on $\{\boldsymbol{P}\}$, with the continuity and monotonity properties required by Axiom A2. Secondly, it connects the s.l.t. on $\boldsymbol{O}$ with the s.l.t. on $\boldsymbol{P}$ in a manner which reflects the physical picture of universal evenness of time flow on photons and particles. A photon $P^{*}$ satisfying the condition (3) of $\mathrm{A} 9$ is said to be synchronized with $\boldsymbol{O}$.

The configuration $(\boldsymbol{O}, \boldsymbol{P})$ consisting of a standard particle $\boldsymbol{O}$ and a synchronized photon $\boldsymbol{P}$ which intersects $\boldsymbol{O}$ will be called a base of reference. It determines uniquely the pseudoplane $\mathfrak{B}(\boldsymbol{O}, \boldsymbol{P}) \in \mathscr{V}(\boldsymbol{O})$ and two conjugate families of standard photons $\boldsymbol{P}_{\mu}, \boldsymbol{P}_{\nu}^{*}, \mu, v \in \mathfrak{I}$, contained in $\mathfrak{B}(\boldsymbol{O}, \boldsymbol{P})$ and its conjugate respectively and characterized by the following properties: in $O(\nu)$.

(i) $\boldsymbol{P}_{\mu} \in \mathfrak{B}(\boldsymbol{O}, \boldsymbol{P})$ intersects $\boldsymbol{O}$ in $\boldsymbol{O}(u), \boldsymbol{P}_{\nu}^{*} \in \mathfrak{B}^{*}(\boldsymbol{O}, \boldsymbol{P})$ intersects $\boldsymbol{O}$ 
(ii) $\boldsymbol{P}_{\mu}, \boldsymbol{P}_{\nu}^{*}$ are both synchronized with $\boldsymbol{O}$. one $\boldsymbol{P}_{v}^{*}$.

(iii) Through every $q \in|\mathfrak{B}(\boldsymbol{O}, \boldsymbol{P})|$ there goes exactly one $\boldsymbol{P}_{\mu}$ and exactly

(i) follows from A6 which asserts that through each $O(\mu)$ there goes exactly one $\left\{\boldsymbol{P}_{\mu}\right\}$ which intersects $\boldsymbol{P}_{\nu}^{*}(\boldsymbol{v} \neq \mu)$ and hence is in $\mathfrak{B}(\boldsymbol{O}, \boldsymbol{P})$, and from Theorem 4 which asserts that each $\boldsymbol{P}_{\nu}^{*}$ yields the same $\left\{\boldsymbol{P}_{\mu}\right\}$. (ii) follows from A9, and (iii) from A4. We call the system of photons $\left[\boldsymbol{P}_{\mu}, \boldsymbol{P}_{\boldsymbol{v}}^{*}\right]$ the reference grid determined by $\boldsymbol{O}$ and $\mathfrak{B}=\mathfrak{B}(\boldsymbol{O}, \boldsymbol{P})$, and denote it by $\Gamma(\boldsymbol{O}, \mathfrak{B})$. The notation assumes that $\boldsymbol{O}$ is standard and $\mathfrak{B} \in \mathscr{V}(\boldsymbol{O})$. Each $q \in \mathfrak{B}$ is uniquely characterized by its grid coordinates $(\mu, v)$ relative to $\Gamma(\boldsymbol{O}, \mathfrak{B})$. Note that if $\mathfrak{B}^{*}$ is conjugate to $\mathfrak{B}$ then the grid coordinates of the same event $q$ relative to $\Gamma\left(\boldsymbol{O}, \mathfrak{V}^{*}\right)$ are $(v, \mu)$. The synchronized time of $q$ relative to $\boldsymbol{O}$ and its synchronized distance from $\boldsymbol{O}$ are expressed in terms of the grid coordinates by

$$
t(O, q)=\frac{1}{2}(\mu+\nu), d(O, q)=\frac{1}{2}|\mu-\nu| .
$$

Axiom A9 has several important implications.

THEOREM 6 (Second law of inertia of particles). Let $\boldsymbol{O}$ be a standard particle, $\mathfrak{B}=\mathfrak{B}(\boldsymbol{O}, \boldsymbol{P}) \in \mathscr{V}(\boldsymbol{O})$, and $\boldsymbol{O}^{\prime}$ a standard particle contained in $|\mathfrak{B}|$. Let $\mu\left(t^{\prime}\right), v\left(t^{\prime}\right)$ be the coordinate of $\boldsymbol{O}^{\prime}\left(t^{\prime}\right)$ relative to $\Gamma(\boldsymbol{O}, \mathfrak{B})$. Then

$$
\mu\left(t^{\prime}\right)=\alpha t^{\prime}+\rho, \nu\left(t^{\prime}\right)=\beta t^{\prime}+\sigma
$$

for suitable real constants $\alpha>0, \beta>0, \rho, \sigma$.

Conversely, the mapping $p=\boldsymbol{O}^{\prime}\left(t^{\prime}\right)$ of $\mathfrak{I}$ into $|\mathfrak{B}|$ where $\mu\left(t^{\prime}\right), \nu\left(t^{\prime}\right)$ given by $(5)$ are the coordinates of $p$ relative to $\Gamma(O, \mathfrak{B})$, is a standard particle.

Proof. Suppose that for some fixed $\mu, \boldsymbol{P}_{\mu}$ intersects $\boldsymbol{O}^{\prime}$, at $\boldsymbol{O}^{\prime}\left(t_{0}^{\prime}\right)$. For arbitrary $\boldsymbol{v}$, let $\left|\boldsymbol{P}_{\nu}^{*}\right| \cap\left|\boldsymbol{O}^{\prime}\right|=\boldsymbol{O}^{\prime}\left(t^{\prime}\right)$. Since $\boldsymbol{P}_{\mu}$ is synchronized with $\boldsymbol{O}, q=\left|\boldsymbol{P}_{\mu}\right| \cap\left|\boldsymbol{P}_{\nu}^{*}\right|=\boldsymbol{P}_{\mu}\left(\frac{1}{2}(u+v)\right)$. If $\boldsymbol{P}_{\mu}^{\prime}$ is homographic with $\boldsymbol{P}_{\mu}$ and synchronized with $\boldsymbol{O}^{\prime}$ then we have for the same event $q=\boldsymbol{P}_{\mu}^{\prime}\left(\frac{1}{2}\left(t^{\prime}+t_{0}^{\prime}\right)\right)$. It follows from $\mathrm{A} 8$ and $\mathrm{A} 9$, since $\mu$ and $t_{0}^{\prime}$ are fixed, that $\nu\left(t^{\prime}\right)=\beta t^{\prime}+\sigma$ for suitable $\beta>0, \sigma$, and the constants are necessarily independent of $\mu$. The proof of $\mu\left(t^{\prime}\right)=\alpha t^{\prime}+\rho$ is obtained similarly, by considering a fixed $\boldsymbol{P}_{\nu}^{*}$ and variable $\boldsymbol{P}_{\mu}$.

Conversely, let $\mu_{i}=\alpha t_{i}+\rho, \nu_{i}=\beta t_{i}+\sigma, i=1,2$, with $t_{1}<t_{2}$, then $\mu_{1}<\mu_{2}$ and $\nu_{1}<\nu_{2}$. Hence if $p_{i}$ is the event with coordinates $\left(\mu_{i}, \nu_{i}\right)$ and $q=\left|\boldsymbol{P}_{\boldsymbol{v}_{1}}^{*}\right| \cap\left|\boldsymbol{P}_{\mu_{2}}\right|$ then $q=\boldsymbol{P}_{\boldsymbol{\nu}_{1}}^{*}\left(\frac{1}{2}\left(\boldsymbol{v}_{1}+u_{2}\right)\right)=\boldsymbol{P}_{\mu_{2}}\left(\frac{1}{2}\left(v_{1}+\mu_{2}\right)\right)$, $p_{1}=\boldsymbol{P}_{\nu_{1}}^{*}\left(\frac{1}{2}\left(v_{1}+\mu_{1}\right)\right), p_{2}=\boldsymbol{P}_{\mu_{2}}\left(\frac{1}{2}\left(v_{2}+\mu_{2}\right)\right)$ by the synchronization condition, hence $p_{1}<q<p_{2}$, and there is an observer $\left\{\boldsymbol{O}^{\prime}\right\}$ through $p_{1}$ and $p_{2}$ by A5. By the first half of Theorem 6, the equations of a suitable standard 1epresentative $\boldsymbol{O}^{\prime}$ are given by (5), and the proof of the theorem is complete.

The Theorem expresses the fact that the particle $\boldsymbol{O}^{\prime}$ moves with 
constant speed relative to $\boldsymbol{O}$. To verify this and to calculate the speed, let $p_{i}=\boldsymbol{O}^{\prime}\left(t_{i}\right), i=1,2$, be events on $\boldsymbol{O}^{\prime}$ with $t_{1}<t_{2}$ and grid coordinates $\left(\mu_{i}, v_{i}\right)$; then by (4) and (5).

$$
\begin{aligned}
\left|d\left(\boldsymbol{O}, p_{2}\right)-d\left(\boldsymbol{O}, p_{1}\right)\right| & =\frac{1}{2}|\alpha-\beta|\left(t_{2}-t_{1}\right), \\
\left|t\left(\boldsymbol{O}, p_{2}\right)-t\left(\boldsymbol{O}, p_{1}\right)\right| & =\frac{1}{2}(\alpha+\beta)\left(t_{2}-t_{1}\right),
\end{aligned}
$$

and the relative speed.

$$
v\left(\boldsymbol{O}^{\prime}, \boldsymbol{O}\right)=\left|\frac{d\left(\boldsymbol{O}, p_{2}\right)-d\left(\boldsymbol{O}, p_{1}\right)}{t\left(\boldsymbol{O}, p_{2}\right)-t\left(\boldsymbol{O}, p_{1}\right)}\right|=\frac{|\alpha-\beta|}{\alpha+\beta}
$$

is independent of $t_{1}$ and $t_{\mathbf{2}}$.

Since $\alpha>0, \beta>0$, we always have

$$
0 \leqq v\left(\boldsymbol{O}^{\prime}, \boldsymbol{O}\right)<\mathbf{1}
$$

$v\left(\boldsymbol{O}^{\prime}, \boldsymbol{O}\right)=0$ if and only if $\alpha=\beta$. In that case either $\boldsymbol{O}^{\prime}$ is homographic with $\boldsymbol{O}$, namely if also $\rho=\sigma$, or $\boldsymbol{O}$ and $\boldsymbol{O}^{\prime}$ have no event in common; for events on $\boldsymbol{O}$ have grid coordinates $\boldsymbol{\nu}=\mu$.

We say that $\left\{\boldsymbol{O}^{\prime}\right\}$ is parallel to $\{\boldsymbol{O}\}$ and write $\left\{\boldsymbol{O}^{\prime}\right\} \mid\{\boldsymbol{O}\}$ iff $\boldsymbol{O}^{\prime}$ is in scme $\mathfrak{B} \in \mathscr{V}(\boldsymbol{O})$ and $\alpha=\beta$ in (5). Necessary and sufficient for $\alpha=\beta$ is that either $\left\{\boldsymbol{O}^{\prime}\right\}=\{\boldsymbol{O}\}$ or that $\boldsymbol{O}^{\prime}$ and $\boldsymbol{O}$ do not intersect; therefore $\{\boldsymbol{O}\} \mid\{\boldsymbol{O}\}$, and $\left\{\boldsymbol{O}^{\prime}\right\}|\{\boldsymbol{O}\} \Rightarrow\{\boldsymbol{O}\}|\left\{\boldsymbol{O}^{\prime}\right\}$. We shall find later that also parallelity of observers is an equivalence relation.

If the local time on $O^{\prime}\left(t^{\prime}\right)$ is transformed by $t^{\prime}=1 / \alpha\left(t^{\prime \prime}+\frac{1}{2}(\sigma-\rho)\right)$ then the equation of $O^{\prime \prime}\left(t^{\prime \prime}\right)=O^{\prime}\left(t^{\prime}\right)$ becomes $\mu\left(t^{\prime \prime}\right)=t^{\prime \prime}+\frac{1}{2}(\rho+\sigma)$, $v\left(t^{\prime \prime}\right)=t^{\prime \prime}-\frac{1}{2}(\rho+\sigma)$. Thus every $\left\{\boldsymbol{O}^{\prime}\right\}$ which is parallel to $\{\boldsymbol{O}\}$ has a unique standard representative $\boldsymbol{O}^{\prime}\left(t^{\prime}\right)$ whose equation relative to $\Gamma(\boldsymbol{O}, \mathfrak{B})$ is in the normal form

$$
\mu\left(t^{\prime}\right)=t^{\prime}+\delta, \nu\left(t^{\prime}\right)=t^{\prime}-\delta .
$$

We then say that $\boldsymbol{O}^{\prime}$ is synchronized with $\boldsymbol{O}$ and write $\boldsymbol{O}^{\prime} \| \boldsymbol{O}$; in fact $t^{\prime}=\frac{1}{2}\left(\mu\left(t^{\prime}\right)+v\left(t^{\prime}\right)\right)$ is just the synchronized time of the event $\left(\mu\left(t^{\prime}\right), v\left(t^{\prime}\right)\right)$ relative to $O$, and $|\delta|=\frac{1}{2}\left|\mu\left(t^{\prime}\right)-v\left(t^{\prime}\right)\right|$ is the synchronized distance of the event from $\boldsymbol{O}$. Thus all events of $\boldsymbol{O}^{\prime}$ are at the same distance $|\delta|$ from $\boldsymbol{O}$; we denote it by $d\left(\boldsymbol{O}^{\prime}, \boldsymbol{O}\right)$.

Theorem 7. Given $\boldsymbol{O} \in \mathcal{O}^{*}$ and $q \in \mathfrak{M}$, there is precisely one $\boldsymbol{O}^{\prime} \in \mathcal{O}^{*}$ such that $q \in\left|\boldsymbol{O}^{\prime}\right|$ and $\boldsymbol{O}^{\prime} \| \boldsymbol{O}$.

Proof. If $q \in|\boldsymbol{O}|$ then we must have $\boldsymbol{O}^{\prime}=\boldsymbol{O}$. If $q \notin|\boldsymbol{O}|$ then $q$ is a uniquely determined pseudoplane $|\mathfrak{B}|, \mathfrak{B} \in \mathscr{V}(\boldsymbol{O})$ and the statement follows from the fact that for given $\mu, \nu$, equation (8) can be solved uniquely for $\delta$.

We shall need the transformation equations of grid coordinates from $\Gamma(\boldsymbol{O}, \mathfrak{B})$ to $\Gamma\left(\boldsymbol{O}^{\prime}, \mathfrak{B}\right)$ where $\left|\boldsymbol{O}^{\prime}\right| \subset|\mathfrak{B}|$ and $\boldsymbol{O}^{\prime}$ is given by $(5)$. They are 
obtained from (5) by writing $t^{\prime}=\mu^{\prime}$ in the first equation and $t^{\prime}=\nu^{\prime}$ in the second:

$$
\mu=\alpha \mu^{\prime}+\rho, \nu=\beta v^{\prime}+\sigma .
$$

If in particular $\boldsymbol{O}^{\prime} \| \boldsymbol{O}$ then the equations are

$$
\mu=\mu^{\prime}+\delta, \nu=v^{\prime}-\delta .
$$

It follows from here that

$$
|\boldsymbol{\delta}|=d\left(\boldsymbol{O}^{\prime}, \boldsymbol{O}\right)=d\left(\boldsymbol{O}, \boldsymbol{O}^{\prime}\right) .
$$

Theorem 8. For every $O \in \mathcal{O}^{*}, O \| O$. Furthermore $O^{\prime}\|O \Rightarrow O\| O^{\prime}$.

Proof. The first statement is trivial. The second statement follows from the fact, seen from (10), that the event $O(t)$ has coordinates $\mu^{\prime}=t-\delta$, $v^{\prime}=t+\delta$ relative to $\Gamma\left(\boldsymbol{O}^{\prime}, \mathfrak{B}\right)$.

\section{Al0 (AXIOM OF INERTIAL SYSTEMS)}

Let $\boldsymbol{O}^{\prime} \| \boldsymbol{O}$. Then $t\left(\boldsymbol{O}^{\prime}, q\right)=t(\boldsymbol{O}, q)$ for every $q \in \mathfrak{W}$.

The axiom expresses the fact that two observers who are at relative rest and who have synchronized their clocks are in agreement when they extend their time reckoning to the whole of the Universe. It is due to this axiom, which seems to be independent of the others and which is the relativistic equivalent of Newton's principle of "absolute time", that we are allowed to speak of an inertial system relative to a standard observer.

An important consequence of the axiom is

THEOREM 9. $O \| O^{\prime}$ is an equivalence relation.

We have already seen that the relation is reflexive and symmetric (Theorem 8). We have to show that $\boldsymbol{O} \| \boldsymbol{O}^{\prime}, \boldsymbol{O}|\boldsymbol{O}|^{\prime \prime}$ imply $\boldsymbol{O}^{\prime} \| \boldsymbol{O}^{\prime \prime}$. Now the first two conditions imply, by Al0, that $t\left(\boldsymbol{O}^{\prime}, q\right)=t\left(\boldsymbol{O}^{\prime \prime}, q\right)$ for every $q \in \mathfrak{B}$. The theorem therefore follows from the following converse of Al0:

Theorem 10. If $t(\boldsymbol{O}, q)=t\left(\boldsymbol{O}^{\prime}, q\right)$ for every $q \in \mathfrak{W}$ then $\boldsymbol{O} \| \boldsymbol{O}^{\prime}$.

Proof. Let $q_{1} \in\left|\boldsymbol{O}^{\prime}\right|$ and $\boldsymbol{O}^{\prime \prime}|| \boldsymbol{O}, q_{\mathbf{1}} \in\left|\boldsymbol{O}^{\prime \prime}\right|$. If $\boldsymbol{O}^{\prime}=\boldsymbol{O}^{\prime \prime}$ then we are finished, otherwise $\left|\boldsymbol{O}^{\prime \prime}\right| \subset\left|\mathfrak{B}^{\prime}\right|$ for some $\mathfrak{B}^{\prime} \in \mathfrak{B}\left(\boldsymbol{O}^{\prime}\right)$. ByA $10, t(\boldsymbol{O}, q)=t\left(\boldsymbol{O}^{\prime \prime}, q\right)$ for all $q \in \mathfrak{B}$, hence $t\left(\boldsymbol{O}^{\prime}, q\right)=t\left(\boldsymbol{O}^{\prime \prime}, q\right)$ for every $q \in\left|\mathfrak{B}^{\prime}\right|$. But from transformation equations (9) (with $\mu^{\prime \prime}, \nu^{\prime \prime}$ replacing $\mu, v$ ) it is seen that $\frac{1}{2}\left(\mu^{\prime}+v^{\prime}\right)=\frac{1}{2}\left(\mu^{\prime \prime}+v^{\prime \prime}\right)$ for all $\mu^{\prime}, v^{\prime}$ if and only if $\alpha=\beta=1$ and $\rho+\sigma=0$ in (9), hence $\boldsymbol{O}^{\prime}|| \boldsymbol{O}^{\prime \prime}$. But $q_{1} \in\left|\boldsymbol{O}^{\prime}\right| \cap\left|\boldsymbol{O}^{\prime \prime}\right|$, therefore $\boldsymbol{O}^{\prime}=\boldsymbol{O}^{\prime \prime}, \boldsymbol{O}^{\prime} \| \boldsymbol{O}$.

\section{All (AXIOM OF PARALlelity)}

Let $(\boldsymbol{O}, \boldsymbol{P})$ and $\left(\boldsymbol{O}^{\prime}, \boldsymbol{P}^{\prime}\right)$ be bases of reference such that $\{\boldsymbol{O}\}\left|\left\{\boldsymbol{O}^{\prime}\right\},\{\boldsymbol{P}\}\right|\left\{\boldsymbol{P}^{\prime}\right\}$. Let $\left\{\boldsymbol{P}^{*}\right\}$ be a l.s. in $\mathfrak{B}(\boldsymbol{O}, \boldsymbol{P})$, conjugate to $\boldsymbol{P}$, and $\left\{\boldsymbol{P}^{* *}\right\}$ a l.s. in $\mathfrak{B}\left(\boldsymbol{O}^{\prime}, \boldsymbol{P}^{\prime}\right\}$, conjugate to $\boldsymbol{P}^{\prime}$. Then $\left\{\boldsymbol{P}^{*}\right\} \mid\left\{\boldsymbol{P}^{*}\right\}$. 
The axiom is in a sense a dual of Alo for photons, as seen from Theorem 11 below. It attributes an objective meaning to the notion that two light signals emitted by observers at relative rest travel in the same direction.

THEOREM 11. $\{\boldsymbol{P}\} \mid\left\{\boldsymbol{P}^{\prime}\right\}$ is an equivalence relation.

We have to show that $\{\boldsymbol{P}\}\left|\left\{\boldsymbol{P}^{\prime}\right\},\{\boldsymbol{P}\}\right|\left\{\boldsymbol{P}^{\prime \prime}\right\}$ imply $\left\{\boldsymbol{P}^{\prime}\right\} \mid\left\{\boldsymbol{P}^{\prime \prime}\right\}$. Now if $\{\boldsymbol{P}\} \mid\left\{\boldsymbol{P}^{\prime}\right\}$ then there is an $\boldsymbol{O}^{\prime} \in \mathcal{O}, \boldsymbol{P}^{\prime *} \in \mathscr{P}$ such that both $\boldsymbol{P}$ and $\boldsymbol{P}^{\prime}$ are in $\mathfrak{B}^{*}\left(\boldsymbol{O}^{\prime}, \boldsymbol{P}^{* *}\right)$ and are conjugate to $\boldsymbol{P}^{\prime *}$. Let $\{\boldsymbol{P}\}\left|\left\{\boldsymbol{P}^{\prime}\right\}, q \in\right| \boldsymbol{P}^{\prime \prime} \mid$, and let $\left\{\boldsymbol{O}^{\prime \prime}\right\}\left|\left\{\boldsymbol{O}^{\prime}\right\}, q \in\right| \boldsymbol{O}^{\prime \prime} \mid$ such that $\left(\boldsymbol{O}^{\prime \prime}, \boldsymbol{P}^{\prime \prime}\right)$ is a base of reference. Let $\left\{\boldsymbol{P}^{*}\right\}$ be conjugate to $\left\{\boldsymbol{P}^{\prime \prime}\right\}$ in $\mathfrak{B}\left(\boldsymbol{O}^{\prime \prime}, \boldsymbol{P}^{\prime \prime}\right)$ then by All, $\left\{\boldsymbol{P}^{*}\right\} \mid\left\{\boldsymbol{P}^{\prime *}\right\}$ and since $\left\{\boldsymbol{P}^{\prime \prime}\right\}$ is conjugate to $\left\{\boldsymbol{P}^{*}\right\}$ and $\left\{\boldsymbol{P}^{\prime}\right\}$ is conjugate to $\left\{\boldsymbol{P}^{*}\right\}$, again by All we have $\left\{\boldsymbol{P}^{\prime}\right\} \mid\left\{\boldsymbol{P}^{\prime \prime}\right\}$.

A class of parallel light signals is called a direction. Two pseudo planes $\mathfrak{B}(\boldsymbol{O}, \boldsymbol{P})$ and $\mathfrak{B}\left(\boldsymbol{O}^{\prime}, \boldsymbol{P}^{\prime}\right)$ are called parallel and denoted $\mathfrak{B}(\boldsymbol{O}, \boldsymbol{P}) \mid \mathfrak{B}\left(\boldsymbol{O}^{\prime}, \boldsymbol{P}^{\prime}\right)$ iff $\{\boldsymbol{O}\} \mid\left\{\boldsymbol{O}^{\prime}\right\}$ and $\left\{\boldsymbol{P}\left\{\mid\left\{\boldsymbol{P}^{\prime}\right\}\right.\right.$. Axiom All asserts that the definition is independent of the bases of reference selected in $\mathfrak{B}(\boldsymbol{O}, \boldsymbol{P})$ or $\mathfrak{B}\left(\boldsymbol{O}^{\prime}, \boldsymbol{P}^{\prime}\right)$. From Theorems 9 and 11 it follows that

THEOREM 12. $\mathfrak{B}(\boldsymbol{O}, \boldsymbol{P}) \mid \mathfrak{B}\left(\boldsymbol{O}^{\prime}, \boldsymbol{P}^{\prime}\right)$ is an equivalence relation.

From Axiom A7 and Theorem 7 it follows that

Theorem 13. Given $\mathfrak{B}(\boldsymbol{O}, \boldsymbol{P})$ and an event $q$ there is exactly one $\mathfrak{B}\left(\boldsymbol{O}^{\prime}, \boldsymbol{P}^{\prime}\right)$ through $q$ which is parallel to $\mathfrak{B}(\boldsymbol{O}, \boldsymbol{P})$.

If $\mathfrak{B}(\boldsymbol{O}, \boldsymbol{P}) \mid \mathfrak{B}^{*}\left(\boldsymbol{O}^{\prime}, \boldsymbol{P}^{\prime}\right)$ then we call $\mathfrak{B}(\boldsymbol{O}, \boldsymbol{P})$ and $\mathfrak{B}\left(\boldsymbol{O}^{\prime}, \boldsymbol{P}^{\prime}\right)$ antiparallel.

\section{Relativistic kinematics}

We are now in a position to derive the laws of relativistic kinematics when motion is restricted to one space direction. We have already obtained (formula (7)) an expression for the relative speeds of observers in terms of the grid coordinates. We now prove

THEOREM 14. The relative speed $v\left(\boldsymbol{O}^{\prime}, \boldsymbol{O}\right)$ is independent of the local time on $\boldsymbol{O}$ and $\boldsymbol{O}^{\prime}$. Furthermore

$$
v\left(\boldsymbol{O}, \boldsymbol{O}^{\prime}\right)=v\left(\boldsymbol{O}^{\prime}, \boldsymbol{O}\right) .
$$

This is Urquhart's theorem mentioned in the introduction. Speed of course is always expressed as a fraction of the speed of light.

First, it is clear that expression (7) only depends on the events through which $\boldsymbol{O}^{\prime}$ passes, but not on the local time on $\boldsymbol{O}^{\prime}$. If the s.l.t. is changed on $O$ by a linear transformation (2) then both $\alpha$ and $\beta$ receive the same factor $\gamma$, and (7) remains unchanged. Thus we only have to verify (12).

But we have already seen in (9) that the transformation to grid coordinates relative to $\boldsymbol{O}^{\prime}$, are given by $\mu=\alpha \mu^{\prime}+\rho, v=\beta \nu^{\prime}+\sigma$, hence 
with

$$
\mu^{\prime}=\alpha^{\prime} \mu+\rho^{\prime}, \nu^{\prime}=\beta^{\prime} \nu+\sigma
$$

$$
\alpha^{\prime}=1 / \alpha, \beta^{\prime}=1 / \beta, \rho^{\prime}=-\rho / \alpha, \sigma^{\prime}=-\sigma / \beta,
$$

and

as required.

$$
v\left(\boldsymbol{O}, \boldsymbol{O}^{\prime}\right)=\frac{\left|\alpha^{\prime}-\beta^{\prime}\right|}{\alpha^{\prime}+\beta^{\prime}}=\frac{|\beta-\alpha|}{\beta+\alpha}=v\left(\boldsymbol{O}^{\prime}, \boldsymbol{O}\right),
$$

Next we derive the composition rule of relative speeds. Let $\left\{\boldsymbol{O}_{1}\right\},\left\{\boldsymbol{O}_{2}\right\}$ be observers in $|\mathfrak{B}(\boldsymbol{O}, \boldsymbol{P})|$ and $(\lambda, \mu)$ the coordinates reltive to $\Gamma(\boldsymbol{O}, \mathfrak{B})$,

$$
\mu_{i}=\alpha_{i} t_{i}+\rho_{i}, \nu_{i}=\beta_{i} t_{i}+\sigma_{i}
$$

the equations of $\boldsymbol{O}_{i}, i=1,2$. Transforming to coordinates $\left(\lambda^{\prime}, \mu^{\prime}\right)$ relative to $\Gamma\left(\boldsymbol{O}_{1}, \mathfrak{B}\right)$, we obtain from $(9)$

$$
\mu=\alpha_{1} \mu^{\prime}+\rho_{1}, \nu=\beta_{1} \nu^{\prime}+\sigma_{1},
$$

and substituting into (14) with $i=2$ we obtain

hence

$$
\mu_{2}^{\prime}=\frac{1}{\alpha_{1}}\left(\alpha_{2} t_{2}+\rho_{2}-\rho_{1}\right), v_{2}^{\prime}=\frac{1}{\beta_{1}}\left(\beta_{2} t_{2}+\sigma_{2}-\sigma_{1}\right),
$$

Hence if we set

$$
v\left(O_{1}, O_{2}\right)=\frac{\left|\alpha_{2} / \alpha_{1}-\beta_{2}\right| \beta_{1} \mid}{\alpha_{2} / \alpha_{1}+\beta_{2} / \beta_{1}}=\frac{\left|\alpha_{2} / \beta_{2}-\alpha_{1} / \beta_{1}\right|}{\alpha_{2} / \beta_{2}+\alpha_{1} / \beta_{1}}
$$

$$
v_{1}=\frac{\alpha_{1} / \beta_{1}-1}{\alpha_{1} / \beta_{1}+1}, \quad v_{2}=\frac{\alpha_{2} / \beta_{2}-1}{\alpha_{2} / \beta_{2}+1}
$$

so that $v\left(\boldsymbol{O}, \boldsymbol{O}_{1}\right)=\left|v_{1}\right|, v\left(\boldsymbol{O}, \boldsymbol{O}_{2}\right)=\left|v_{2}\right|$, we obtain

$$
v\left(\boldsymbol{O}_{1}, \boldsymbol{O}_{2}\right)=\frac{\left|v_{2}-v_{1}\right|}{I-v_{1} v_{2}}
$$

which is the relativistic composition rule of relative speeds.

To derive the formula for the Lorentz-Fitzgerald contraction, we have to generalize the definition of distance. The proof proceeds on familiar lines. Given $\boldsymbol{O} \in \mathcal{O}^{*}$, let $p_{1}, p_{2}$ be distinct events in $\mathfrak{W}, \boldsymbol{O}_{1}, \boldsymbol{O}_{2}$ particles such that

$$
p_{1} \in\left|O_{1}\right|, p_{2} \in\left|O_{2}\right|, O_{1}|| O, O_{2}|| O \text {. }
$$

Then the distance $d\left(\boldsymbol{O} ; p_{1}, p_{2}\right)$ of $p_{1}$ and $p_{2}$ relative to $\boldsymbol{O}$ is defined by the formula

$$
d\left(\boldsymbol{O} ; p_{1}, p_{2}\right)=d\left(\boldsymbol{O}_{1}, \boldsymbol{O}_{2}\right)
$$

The definition is in accordance with Einstein's definition of distance by 
means of reflected light signals. The particles $\boldsymbol{O}_{1}, \boldsymbol{O}_{2}$ themselves represent the endpoints of a "rigid" measuring rod.

Let $\mathfrak{B}$ be the pseudoplane determined by $\boldsymbol{O}_{1}, \boldsymbol{O}_{2}$ and $\left\{\boldsymbol{O}^{\prime}\right\}$ any observer in $\mathfrak{B}$. Suppose the events $p_{1} \in\left|\boldsymbol{O}_{1}\right|, p_{2} \in\left|\boldsymbol{O}_{2}\right|$ are simultaneous relative to $\boldsymbol{O}^{\prime}$, i.e.

$$
t\left(\boldsymbol{O}^{\prime}, p_{1}\right)=t\left(\boldsymbol{O}^{\prime}, p_{2}\right)
$$

If $(\lambda, \mu)$ are grid coordinates relative to $\boldsymbol{O}_{1},\left(\lambda^{\prime}, \mu^{\prime}\right)$ relative to $\boldsymbol{O}^{\prime}$ then we have

$$
\mu=\alpha t^{\prime}+\rho, \nu=\beta t^{\prime}+\sigma
$$

for the equation of $\boldsymbol{O}^{\prime}$ relative to $\Gamma\left(\boldsymbol{O}_{1}, \mathfrak{B}\right)$, and

$$
\mu=\alpha \mu^{\prime}+\rho, v=\beta v^{\prime}+\sigma
$$

for the transformation equations of the grid coordinates.

Let $p_{1}$ have coordinates $\left(\mu_{1}, \mu_{1}\right), p_{2}$ have coordinates $\left(\mu_{2}+\delta, \mu_{2}-\delta\right)$ relative to $\Gamma\left(\boldsymbol{O}_{1}, \mathfrak{B}\right)$ where $|\delta|=d\left(\boldsymbol{O}_{1}, \boldsymbol{O}_{2}\right)$. Hence if $\left(\mu_{i}^{\prime}, \boldsymbol{v}_{i}^{\prime}\right)$ are the coordinates of $p_{i}$ relative to $\Gamma\left(\boldsymbol{O}^{\prime}, \mathfrak{B}\right)$ then we get

$$
\mu_{1}=\alpha \mu_{1}^{\prime}+\rho=\beta v_{1}^{\prime}+\sigma, \mu_{2}+\delta=\alpha \mu_{2}^{\prime}+\rho, \nu_{2}-\delta=\beta v_{2}^{\prime}+\sigma,
$$

and from the conditions of simultaneity $\mu_{1}^{\prime}+\nu_{1}^{\prime}=\mu_{2}^{\prime}+v_{2}^{\prime}$. These together give

and

$$
\left(\mu_{1}-\mu_{2}\right)(1 / \alpha+1 / \beta)=\delta(1 / \alpha-1 / \beta)
$$

$$
\begin{aligned}
d\left(O^{\prime} ; p_{1}, p_{2}\right) & =\frac{1}{2}\left|\left(\nu_{1}^{\prime}-\mu_{1}^{\prime}\right)-\left(v_{2}^{\prime}-\mu_{2}^{\prime}\right)\right| \\
& =\left|\mu_{2}^{\prime}-\mu_{1}^{\prime}\right|=\frac{2|\delta|}{\alpha+\beta} .
\end{aligned}
$$

This is defined as the distance of $\boldsymbol{O}_{1}$ and $\boldsymbol{O}_{2}$ relative to $\boldsymbol{O}^{\prime}$; we denote it by $d\left(\boldsymbol{O}^{\prime} ; \boldsymbol{O}_{1}, \boldsymbol{O}_{2}\right)$. Its value is independent of the choice of $p_{1}$ and $p_{2}$ on $\boldsymbol{O}_{1}$ and $\boldsymbol{O}_{2}$ provided that they are simultaneous relative to $\boldsymbol{O}^{\prime}$, but depends on the standard local time on $\boldsymbol{O}^{\prime}$.

To obtain an objective measure of distance, it is necessary to synchronize the times on $\boldsymbol{O}$ and $\boldsymbol{O}^{\prime}$. This is accomplished as follows: Let $O_{1}^{\prime}\left\|O^{\prime}, O_{2}^{\prime}\right\| O^{\prime}$ such that

$$
d\left(\boldsymbol{O}_{1}^{\prime}, \boldsymbol{O}_{2}^{\prime}\right)=d\left(\boldsymbol{O}_{1}, \boldsymbol{O}_{2}\right)=|\delta| .
$$

We say that $\boldsymbol{O}$ and $\boldsymbol{O}^{\prime}$ are synchronized iff

$$
d\left(\boldsymbol{O}^{\prime} ; \boldsymbol{O}_{1}, \boldsymbol{O}_{2}\right)=d\left(\boldsymbol{O} ; \boldsymbol{O}_{1}^{\prime}, \boldsymbol{O}_{2}^{\prime}\right)
$$

for any pair of observers $\boldsymbol{O}_{1}\left\|\boldsymbol{O}, \boldsymbol{O}_{2}\right\| \boldsymbol{O}, \boldsymbol{O}_{1}^{\prime}\left\|\boldsymbol{O}^{\prime}, \boldsymbol{O}_{2}^{\prime}\right\| \boldsymbol{O}^{\prime}$ satisfying the condition (17). This is so if and only if 
i.e.

$$
\frac{|\delta|}{\alpha+\beta}=\frac{|\delta|}{1 / \alpha+1 / \beta} \text { for every } \delta
$$

$$
\alpha \beta=1
$$

in the transformation equations from $\boldsymbol{O}$ to $\boldsymbol{O}^{\prime}$. The condition can always be enforced by a suitable change of the standard local time on $\boldsymbol{O}$ or $\boldsymbol{O}^{\prime}$. The definition is in accordance with our previous convention regarding the synchrorization of parallel particles.

By using condition (19) we find for the speed of $\boldsymbol{O}^{\prime}$ relative to $\boldsymbol{O}$

$$
\begin{aligned}
v & =\frac{|\alpha-\beta|}{\alpha+\beta}=\frac{\left|1-\beta^{2}\right|}{1+\beta^{2}} \\
\sqrt{1-v^{2}} & =\frac{2 \beta}{1+\beta^{2}}=\frac{2}{\beta+1 / \beta}
\end{aligned}
$$

hence

$$
d\left(\boldsymbol{O}^{\prime} ; \boldsymbol{O}_{1}, \boldsymbol{O}_{2}\right)=|\delta| \sqrt{1-v^{2}}=d\left(\boldsymbol{O}_{1}, \boldsymbol{O}_{2}\right) \sqrt{1-v^{2}}
$$

The formula expresses the distance of a pair of parallel particles (endpoints of a rigid rod) relative to a moving observer, provided that all relevant times are synchronized; it is the well-known formula for the LorentzFitzgerald contraction.

The reference grid we have been using so far to locate events in a pseudoplane $\mathfrak{B}$ consisted of a system of photons $\left[\boldsymbol{P}_{\mu}, \boldsymbol{P}_{\nu}^{*}\right]$. We pass now to the more conventional method of describing events by time and distance coordinates, given by

$$
x=\frac{1}{2}(\mu-\nu), t=\frac{1}{2}(\mu+\nu) \text {. }
$$

If $\boldsymbol{O}^{\prime}$ is a standard particle on $\mathfrak{B}$, intersecting $\boldsymbol{O}$ at $q$, and if the origin of the local time on $\boldsymbol{O}$ and $\boldsymbol{O}^{\prime}$ is chosen so that $q=\boldsymbol{O}(O)=\boldsymbol{O}^{\prime}(O)$, then the transformation equations to $\Gamma\left(\boldsymbol{O}^{\prime}, \mathfrak{B}\right)$ are $\mu=\alpha \mu^{\prime}, v=\beta v^{\prime}$ where $\mu^{\prime}=x^{\prime}+t^{\prime}, v^{\prime}=t^{\prime}-x^{\prime}$. This gives

$$
\begin{aligned}
x & =\frac{1}{2}(\alpha+\beta) x^{\prime}+\frac{1}{2}(\alpha-\beta) t^{\prime}, \\
t & =\frac{1}{2}(\alpha-\beta) x^{\prime}+\frac{1}{2}(\alpha+\beta) t^{\prime} .
\end{aligned}
$$

If moreover $\boldsymbol{O}^{\prime}$ is synchronized with $\boldsymbol{O}$ and $v$ is the relative speed of $\boldsymbol{O}^{\prime}$ and $O$ so that $\alpha=1 / \beta, \frac{1}{2}(\alpha+\beta)=\frac{1}{2}(\beta+1 / \beta)=1 / \sqrt{1-v^{2}}$, then we obtain the usual form of the Lorentz transformations

$$
x=\gamma\left(x^{\prime} \pm v t^{\prime}\right), t=\gamma\left( \pm v x^{\prime}+t^{\prime}\right),
$$

with $\gamma=1 / \sqrt{1-v^{2}}$. The validity of these equations depends very essentially on the assumption that the local times on $\boldsymbol{O}$ and $\boldsymbol{O}^{\prime}$ are synchronized. 


\section{Axioms of dimension, symmetry and isotropy}

All axioms formulated so far were compatible with a Universe consisting of a single pseudoplane $\mathfrak{B}(\boldsymbol{O}, \boldsymbol{P})$. We need further axioms to extend the geometry to four dimensions.

\section{A12 (AxIOM OF DIMENSION)}

Let $(\boldsymbol{O}, \boldsymbol{P})$ be a base of reference. Then there is an event $q$ not in $\mathfrak{B}(\boldsymbol{O}, \boldsymbol{P})$.

The axion asserts that $\mathfrak{W}$ is at least three dimensional.

A one to one mapping $\varphi$ of $\mathfrak{W}$ onto $\mathfrak{B}$ is called an automorphism iff it preserves standard particles and photons:

$$
\boldsymbol{O} \in \mathcal{O}^{*} \Rightarrow \phi \circ \boldsymbol{O} \in \mathcal{O}^{*}, \boldsymbol{P} \in \mathscr{P}^{*} \Rightarrow \boldsymbol{\phi} \circ \boldsymbol{P} \in \mathscr{P}^{*} \text {. }
$$

Clearly the automorphisms of $\mathfrak{B}$ form a group $\mathfrak{A}$, with identity

$$
\varepsilon: \varepsilon(q)=q \text { for all } q \in \mathfrak{W} \text {. }
$$

THEOREM 15. An automorphism carries pseudoplanes into pseudoplanes and preserves parallelity of world lines and pseudoplanes. More specifically, if $\phi \in \mathscr{Q}$ and $\boldsymbol{O}_{\mathbf{1}} \| \boldsymbol{O}_{\mathbf{2}},\left\{\boldsymbol{P}_{\mathbf{1}}\right\} \mid\left\{\boldsymbol{P}_{\mathbf{2}}\right\}$ then $\left(\phi \circ \boldsymbol{O}_{1}\right) \|\left(\phi \circ \boldsymbol{O}_{\mathbf{2}}\right),\left\{\phi \circ \boldsymbol{P}_{\mathbf{1}}\right\} \mid\left\{\phi \circ \boldsymbol{P}_{2}\right\}$.

For let $(\boldsymbol{O}, \boldsymbol{P})$ be a base of reference, $\mathfrak{B}=\mathfrak{B}(\boldsymbol{O}, \boldsymbol{P}), \Gamma(\boldsymbol{O}, \mathfrak{B})=\left[\boldsymbol{P}_{\mu}, \boldsymbol{P}_{\nu}^{*}\right]$. Then $\phi \circ \boldsymbol{P}_{\lambda}^{*}$ is a photon, intersecting $\phi \circ \boldsymbol{O}$ and $\phi \circ \boldsymbol{P}$ hence contained in $\phi(\mathfrak{B})=\mathfrak{B}(\phi \circ \boldsymbol{O}, \phi \circ \boldsymbol{P})$. It intersects $\phi \circ \boldsymbol{O}$ at $\phi \circ \boldsymbol{O}(v)$ from which follows that $\left\{\phi \circ \boldsymbol{P}_{\mu}, \phi \circ \boldsymbol{P}_{\nu}^{*}\right\}$ is the grid determined by the base of reference $(\phi \circ \boldsymbol{O}$, $\phi \circ \boldsymbol{P})$, and coordinates of $q \in \mathfrak{B}(\boldsymbol{O}, \boldsymbol{P})$ with respect to $\Gamma(\boldsymbol{O}, \mathfrak{B})$ are the same as the coordinates of $\phi(q)$ with respect to $\Gamma(\phi \circ \boldsymbol{O}, \phi(\mathfrak{B}))$. All statements of Theorem 15 now follow trivially.

Translations are examples of non-trivial automorphisms. Let $\boldsymbol{O} \in \mathcal{O}^{*}$ and $\lambda$ be a fixed real number. We define $\tau_{\boldsymbol{o}, \lambda}: \mathfrak{W} \rightarrow \mathfrak{W}$ as follows: Given $q \in \mathfrak{W}$ let $q=\boldsymbol{O}^{\prime}\left(t_{0}\right), \boldsymbol{O}^{\prime} \mid \boldsymbol{O}$. Then

In particular

$$
\tau_{\boldsymbol{o}, \lambda}(q)=\boldsymbol{O}^{\prime}\left(t_{0}+\lambda\right) .
$$

$$
\tau_{\boldsymbol{o}, \lambda} \circ \boldsymbol{O}^{\prime}(t)=\boldsymbol{O}^{\prime}(t+\lambda) \in \mathcal{O}^{*}
$$

so that $\tau_{\boldsymbol{o}, \lambda}$ maps every $\boldsymbol{O}^{\prime} \| \boldsymbol{O}$ into a standard particle, homographic with $\boldsymbol{O}^{\prime}$.

Suppose now that $\left\{\boldsymbol{O}_{1}\right\}$ is not parallel to $\{\boldsymbol{O}\}$ and $q \in\left|\boldsymbol{O}_{1}\right|$. By Theorem 7 there is a unique particle $\boldsymbol{O}^{\prime} \| \boldsymbol{O}$ through $q$ and $\boldsymbol{O}_{1}$ lies in a pseudoplane $\mathfrak{B} \in \mathscr{V}\left(\boldsymbol{O}^{\prime}\right)$. Let the equation of $\boldsymbol{O}_{\mathbf{1}}(t)$ relative to $\Gamma\left(\boldsymbol{O}^{\prime}, \mathfrak{B}\right)$ be

$$
\mu=\alpha t+\rho, \nu=\beta \tau+\sigma .
$$

Then by an easy calculation the equation of $\tau_{o, \lambda} \circ O_{1}(t)$ is $\mu=\alpha t+\rho+\lambda$, $\boldsymbol{\nu}=\beta t+\sigma+\lambda$, hence a standard particle (by Theorem 6), parallel to $\boldsymbol{O}_{\mathbf{1}}$. 
Similarly the photons of the corresponding grid $\left\{\boldsymbol{P}_{\mu}, \boldsymbol{P}_{\nu}^{*}\right\}$ are mapped into

$$
\tau_{\boldsymbol{o}, \lambda} \circ \boldsymbol{P}_{\mu}(t)=\boldsymbol{P}_{\mu+\lambda}(t+\lambda), \tau_{\boldsymbol{o}, \lambda} \circ \boldsymbol{P}_{\nu}^{*}(t)=\boldsymbol{P}_{\nu+\lambda}^{*}(t+\lambda) .
$$

We call $\tau_{\boldsymbol{o}, \lambda}$ a time translation by $\lambda$, in the direction of $\boldsymbol{O}$.

If $\boldsymbol{O} \in \mathcal{O}^{*}, \boldsymbol{P} \in \mathscr{P}^{*}$, and $\lambda$ is a fixed positive real number we define the space translation $\sigma_{\boldsymbol{O}, \boldsymbol{p}, \lambda}$ and the light translation $\boldsymbol{v}_{\boldsymbol{O}, \boldsymbol{p}, \lambda}$ as follows: Given $q \in \mathfrak{W}$, let $\left\langle\boldsymbol{O}^{\prime}, \boldsymbol{P}^{\prime}\right)$ be the unique base of reference such that $q=\left|\boldsymbol{O}^{\prime}\right| \cap\left|\boldsymbol{P}^{\prime}\right|$, $\boldsymbol{O}^{\prime} \| \boldsymbol{O},\left\{\boldsymbol{P}^{\prime}\right\} \mid\{\boldsymbol{P}\}$. Let $\boldsymbol{O}_{1} \| \boldsymbol{O},\left|\boldsymbol{O}_{\mathbf{1}}\right| \subset\left|\mathfrak{B}\left(\boldsymbol{O}^{\prime}, \boldsymbol{P}^{\prime}\right)\right|$ with equation

$$
\mu(t)=t-\lambda, \nu(t)=t+\lambda .
$$

Then if $q=\boldsymbol{O}^{\prime}\left(t_{0}\right)$ we define

$$
\sigma_{\boldsymbol{o}, \boldsymbol{p}, \lambda}(q)=\boldsymbol{O}_{1}\left(t_{0}\right), v_{\boldsymbol{o}, \boldsymbol{p}, \lambda}=O_{1}\left(t_{0}+\lambda\right) .
$$

Thus by (4) and (10), $t(\boldsymbol{O}, q)=t\left(\boldsymbol{O}, \sigma_{\boldsymbol{o}, \boldsymbol{p}, \lambda}(q)\right)$. Clearly

$$
\sigma_{\boldsymbol{o}, \boldsymbol{P}, \lambda}=\nu_{\boldsymbol{o}, \boldsymbol{p}, \lambda} \circ\left(\tau_{\boldsymbol{o}, \lambda}\right)^{-1} \text {. }
$$

To show therefore that $\sigma_{\boldsymbol{o}, \boldsymbol{p}, \lambda}$ carries standard particles and photons into standard particles and photons respectively, it is sufficient to prove that $v_{\boldsymbol{O}, \boldsymbol{P}, \lambda}$ has this property.

Let $\boldsymbol{O}_{\mathbf{1}}$ be a given standard particle, $\mathfrak{B}_{1}=\mathfrak{B}\left(\boldsymbol{O}_{1}, \boldsymbol{P}_{1}\right) \in \mathscr{V}\left(\boldsymbol{O}_{\mathbf{1}}\right)$ such that $\left\{\boldsymbol{P}_{1}\right\} \mid\{\boldsymbol{P}\}$. By Theorem 11, $\left\{\boldsymbol{P}_{\mu}\right\} \mid\{\boldsymbol{P}\}$ for every $\boldsymbol{P}_{\mu}$ in $\left.\Gamma \mid \boldsymbol{O}_{1}, \mathfrak{B}_{\mathbf{1}}\right)=\left[\boldsymbol{P}_{\mu}, \boldsymbol{P}_{\nu}^{*}\right]$. By A9 we can choose the s.l.t. on $\left\{\boldsymbol{O}_{1}\right\}^{*}$ so that each $\boldsymbol{P}_{\mu}$ should be synchronized with $\boldsymbol{P}$. Then with respect to this grid of reference the equation of $v_{o, p, 2} \circ O_{1}(t)$ is given by

$$
\mu(t)=t, v(t)=t+2 \lambda,
$$

hence it is a particle parallel to $\boldsymbol{O}_{\mathbf{1}}$.

Next consider a standard photon $\boldsymbol{P}_{\mathbf{1}}$. If $\left\{\boldsymbol{P}_{\mathbf{1}}\right\} \mid\{\boldsymbol{P}\}$ then $\nu_{\boldsymbol{o}, \boldsymbol{p}, \lambda}$ trivially carries $\boldsymbol{P}_{\mathbf{1}}$ into a homographic standard photon. If $\left\{\boldsymbol{P}_{\mathbf{1}}\right\}$ is not parallel to $\{\boldsymbol{P}\}$ then there is a 1.s. $\left\{\boldsymbol{P}^{*}\right\} \mid\{\boldsymbol{P}\}$ intersecting $\boldsymbol{P}_{\mathbf{1}}$ and they determine a pseudoplane $\mathfrak{B}\left(\boldsymbol{O}_{\mathbf{1}}, \boldsymbol{P}_{\mathbf{1}}\right)$ in which every $\left\{\boldsymbol{P}_{\mathbf{1}}^{*}\right\}$ intersecting $\left\{\boldsymbol{P}_{\mathbf{1}}\right\}$ is parallel to $\left\{\boldsymbol{P}^{*}\right\}$ hence (by Theorem 11) to $\{\boldsymbol{P}\}$. By selecting the s.l.t. on $\boldsymbol{P}_{\mathbf{1}}$ as before, we find that $\boldsymbol{\nu}_{\boldsymbol{o}, \boldsymbol{p}, \boldsymbol{i}}$ carries $\boldsymbol{P}_{\mathbf{1}}$ unto a standard photon in $\mathfrak{B}\left(\boldsymbol{O}_{\mathbf{1}}, \boldsymbol{P}_{\mathbf{1}}\right)$, parallel to $\boldsymbol{P}_{1}$.

Since both time and space transalations have inverses $\left(\tau_{\boldsymbol{o}, \lambda}^{-1}=\tau_{\boldsymbol{o},-\lambda}\right.$, $\sigma_{\boldsymbol{o}, \boldsymbol{P}, \lambda}=\sigma_{\boldsymbol{o}, \boldsymbol{P}^{*}, \lambda}$ where $\boldsymbol{P}^{*}$ is a conjugate of $\boldsymbol{P}$ in $\left.\mathfrak{B}(\boldsymbol{O}, \boldsymbol{P})\right)$, they are automorphisms.

We say that the automorphism $\phi \in \mathfrak{A}$ admits $\boldsymbol{O}$ iff $\{\boldsymbol{\phi} \circ \boldsymbol{O}\} \mid\{\boldsymbol{O}\}$; in that case we define $\gamma=\gamma(\phi, \boldsymbol{O})$ by $\boldsymbol{O}^{\prime}(\gamma t+\beta)=\phi \circ \boldsymbol{O}(t)$ where $\boldsymbol{O}^{\prime} \| \boldsymbol{O}$. Hence the symbol $\gamma(\phi, \boldsymbol{O})$ is only defined when $\phi$ admits $\boldsymbol{O}$. 
THEOREM 16. Suppose that the automorphism $\phi \in \mathfrak{A}$ admits $O \in \mathcal{O}^{*}$ and let $\boldsymbol{O}_{\mathbf{1}} \| \boldsymbol{O}$. Then $\phi$ also admits $\boldsymbol{O}_{1}$ and $\gamma\left(\phi, \boldsymbol{O}_{\mathbf{1}}\right)=\gamma(\phi, \boldsymbol{O})$.

Proof. Let $\boldsymbol{O}_{\mathbf{1}}=\boldsymbol{O}_{\mathbf{1}}\left(t_{1}\right)$ be in $\mathfrak{B} \in \mathscr{V}(\boldsymbol{O})$, its equation in $\Gamma(\boldsymbol{O}, \mathfrak{B})=$ $\left[\boldsymbol{P}_{\mu}, \boldsymbol{P}_{\nu}^{*}\right]$ being

$$
\mu\left(t_{1}\right)=t_{1}+\delta, \nu\left(t_{1}\right)=t_{1}-\delta .
$$

Since $\phi$ is an automorphism. $\left[\phi \circ \boldsymbol{P}_{\mu}, \phi \circ \boldsymbol{P}_{\nu}^{*}\right]$ is a grid in $\mathfrak{B}^{\prime}=\phi(\mathfrak{B}) \in \mathscr{V}(\phi \circ \boldsymbol{O})$, by Theorem 15. Let $\boldsymbol{O}^{\prime}\left(t^{\prime}\right)=\phi \circ \boldsymbol{O}(t)$ where $\boldsymbol{O}^{\prime} \| \boldsymbol{O}$, hence $t^{\prime}=\gamma t+\beta, \gamma>0$.

Let $q \in\left|\boldsymbol{O}_{1}\right|$ have coordinates $\mu\left(t_{1}\right), v\left(t_{1}\right)$ in $\Gamma(\boldsymbol{O}, \mathfrak{B})$. Then $q^{\prime}=\phi(q)$ has the same coordinates $(\mu, \nu)$ in $\Gamma\left(\phi \circ O, \mathfrak{B}^{\prime}\right)$ and its coordinates in $\Gamma\left(\boldsymbol{O}^{\prime}, \mathfrak{B}^{\prime}\right)$ are given by $\mu^{\prime}=\gamma \mu+\beta, \nu^{\prime}=\gamma \nu+\beta$. Hence the equation of $\phi \circ \boldsymbol{O}_{1}$ relative to $\Gamma\left(\boldsymbol{O}^{\prime}, \mathfrak{B}^{\prime}\right)$ is

$$
\mu^{\prime}\left(t_{1}\right)=\gamma t_{1}+\gamma \delta+\beta, v^{\prime}\left(t_{1}\right)=\gamma t_{1}-\gamma \delta+\beta
$$

which proves the theorem. It shows furthermore that

$$
d\left(\boldsymbol{O}^{\prime}, q^{\prime}\right)=\gamma|\delta|=\gamma d(\boldsymbol{O}, q) .
$$

Since $q$ was an arbitrary event on $\boldsymbol{O}_{1}$, we conclude:

Corollary. Suppose that $\phi \in \mathfrak{A}$ admits $\boldsymbol{O}$, and $\boldsymbol{O}_{\mathbf{1}} \| \boldsymbol{O}$. Let $\boldsymbol{O}^{\prime}$ be homographic with $\phi \circ O, O_{1}^{\prime}$ homographic with $\phi \circ \boldsymbol{O}_{1}, \boldsymbol{O}^{\prime}\left\|\boldsymbol{O}, \boldsymbol{O}_{1}^{\prime}\right\| \boldsymbol{O}_{1}$. Then

$$
d\left(\boldsymbol{O}^{\prime}, \boldsymbol{O}_{1}^{\prime}\right)=\gamma d\left(\boldsymbol{O}, \boldsymbol{O}_{1}\right) \text {. }
$$

For this reason we call the positive number $\gamma(\phi, O)$ the dilatation ratio of $\phi$ (relative to $\boldsymbol{O}$ ).

We say that the automorphism $\phi$ reflects the base of reference $(\boldsymbol{O}, \boldsymbol{P})$ iff the following is true: $\phi \circ \boldsymbol{O}=\boldsymbol{O}$ (i.e. all events of $\boldsymbol{O}$ are fixed points of $\phi$ ) and $\phi \circ \boldsymbol{P}=\boldsymbol{P}^{*}$ where $\boldsymbol{P}^{*}$ is the (unique) conjugate of $\boldsymbol{P}$ in $\mathfrak{B}(\boldsymbol{O}, \boldsymbol{P})$, synchronized with $\boldsymbol{O}$ and passing through $|\boldsymbol{P}| \cap|\boldsymbol{O}|$.

Theorem 17. If $\phi$ reflects $(\boldsymbol{O}, \boldsymbol{P})$ and $\Gamma(\boldsymbol{O}, \mathfrak{B})=\left[\boldsymbol{P}_{\mu}, \boldsymbol{P}_{\nu}^{*}\right], \mathfrak{B}=\mathfrak{B}(\boldsymbol{O}, \boldsymbol{P})$, then $\phi \circ \boldsymbol{P}_{\mu}=\boldsymbol{P}_{\mu}^{*}, \phi \circ \boldsymbol{P}_{\nu}^{*}=\boldsymbol{P}_{\nu}$ for every $\mu, \nu$.

Hence if $\phi$ reflects $(\boldsymbol{O}, \boldsymbol{P})$ then it reflects every $\left(\boldsymbol{O}, \boldsymbol{P}_{\mu}\right)$ and every $\left(\boldsymbol{O}, \boldsymbol{P}_{\nu}^{*}\right)$. Thus the concept of reflection refers to $\boldsymbol{O}$ and a pair of conjugate pseudoplanes $\mathfrak{B}, \mathfrak{B}^{*} \in \mathscr{V}(\boldsymbol{O})$, and we can more appropriately speak of $\phi$ reflecting $\mathfrak{B}$ (or $\mathfrak{B}^{*}$ ) with respect to $\boldsymbol{O}$. Clearly $\phi$ admits $\boldsymbol{O}$ and the dilatation ratio is 1 ; furthermore, $\phi \circ \phi$ is the identity on $\mathfrak{B}$.

For the proof we may assume that $\boldsymbol{P}=\boldsymbol{P}_{\mathbf{0}}$. Now $\boldsymbol{P}_{\nu}^{*}, \boldsymbol{v} \neq \leq 0$ intersects $P_{0}$ at $P_{0}\left(\frac{1}{2} v\right)=P_{v}^{*}\left(\frac{1}{2} v\right)$ and $O$ at $O(v)=P_{v}^{*}(v)$. But $\phi \circ P_{0}\left(\frac{1}{2} v\right)=P_{0}^{*}\left(\frac{1}{2} v\right)$, $\phi \circ \boldsymbol{O}(v)=\boldsymbol{O}(v)$ therefore $\phi \circ \boldsymbol{P}_{\nu}^{*}$ is a standard photon $\boldsymbol{P}^{\prime}(t)$ with $\boldsymbol{P}^{\prime}\left(\frac{1}{2} v\right)=\boldsymbol{P}_{0}^{*}\left(\frac{1}{2} v\right), \boldsymbol{P}^{\prime}(v)=\boldsymbol{O}(v)$, i.e. identical with $\boldsymbol{P}_{\nu}$. By replacing $\boldsymbol{P}_{0}$ with say $\boldsymbol{P}_{1}^{*}$ in the argument we find similarly that $\phi \circ \boldsymbol{P}_{\mu}=\boldsymbol{P}_{\mu}^{*}$ for all $\mu \neq 1$. 
The missing equations $\phi \circ \boldsymbol{P}_{0}^{*}=\boldsymbol{P}_{\mathbf{0}}$ and $\phi \circ \boldsymbol{P}_{\mathbf{1}}=\boldsymbol{P}_{\mathbf{1}}^{*}$ are found by considering photons intersecting $\boldsymbol{P}_{\frac{1}{2}}$ and $\boldsymbol{O}$, and $\boldsymbol{P}_{\frac{1}{2}}^{*}$ and $\boldsymbol{O}$, respectively.

It follows from the theorem that if $q \in \mathfrak{B}$ has coordinate $(\mu, \nu)$ relative to $\Gamma(O, \mathfrak{B})$ then $\phi(q)$ has coordinates $(v, \mu)$ in $\Gamma(\boldsymbol{O}, \mathfrak{B})$. In particular if $\boldsymbol{O}_{1}|| \boldsymbol{O},\left|\boldsymbol{O}_{\mathbf{i}}\right| \subset|\mathfrak{B}|$, and its equation in $\Gamma(\boldsymbol{O}, \mathfrak{B})$ is

$$
\mu(t)=t+\delta, v(t)=t-\delta .
$$

Hence $\boldsymbol{O}_{1}^{*}$ is obtained from $\boldsymbol{O}_{\mathbf{1}}$ by reflecting it with respect to $\boldsymbol{O}$.

\section{Al3 (AXIOM OF CENTROSYMmetry)}

Given $\boldsymbol{O} \in \mathcal{O}^{*}$ there exists an automorphism $\zeta_{\boldsymbol{o}}$ which reflects every $\mathfrak{B} \in \mathscr{V}(\boldsymbol{O})$ with respect to $\boldsymbol{O}$.

The mapping $\zeta_{\boldsymbol{o}}: \mathfrak{W} \rightarrow \mathfrak{W}$ is uniquely determined by the above condition. In fact $\zeta_{\boldsymbol{o}}(p)=p$ for every $p \in|\boldsymbol{O}|$, and if $q \in \mathfrak{B}, q \notin|\boldsymbol{O}|$ then $\zeta_{\boldsymbol{o}}(q)$ is determined as follows: Let $\mathfrak{B} \in \mathscr{V}(\boldsymbol{O})$ be the unique pseudoplane which contains $q$, and $(\mu, \nu)$ the grid coordinates of $q$ in $\Gamma(O, \mathfrak{B})$. Then $\zeta_{\boldsymbol{o}}(q)$ is in $\mathfrak{B}$ and has grid coordinates $(v, \mu)$. The point in the statement of Al3 is that the mapping so obtained is in fact an automorphism of $\mathfrak{B}$, i.e. carries world lines into world lines. Clearly $\zeta_{\boldsymbol{o}} \circ \zeta_{\boldsymbol{o}}$ is the identity automorphism.

\section{A14 (AXIOM OF REFLECTION SYMMETRY)}

Given $O \in \mathcal{O}^{*}$ and $\mathfrak{B} \in \mathscr{V}(\boldsymbol{O})$, there exists a unique automorphism $\rho=\rho_{\boldsymbol{o}, \mathfrak{V}}$ of $\mathfrak{B}$ with the following property: $\rho$ is a reflection of $\mathfrak{B}$ with respect to $\boldsymbol{O}$ and is a reflection of every $\mathfrak{B}^{\prime} \mid \mathfrak{B}$ with respect to some $\boldsymbol{O}^{\prime}|| \boldsymbol{O},\left|\boldsymbol{O}^{\prime}\right| \subset \mathfrak{B}^{\prime}$.

Axioms Al3 and Al4 are the precise kinematic expressions of what is understood by the centrosymmetry and reflection symmetry of space. We call $\rho_{\boldsymbol{o}, \mathfrak{v}}$ a reflection of $\mathfrak{\mathfrak { B }}$. It determines in every pseudoplane $\mathfrak{B} \mid \mathfrak{B}$ a unique particle $\boldsymbol{O}^{\prime} \| \boldsymbol{O}$ with the property

$$
\rho_{\boldsymbol{o}, \mathfrak{g}} \circ \boldsymbol{O}^{\prime}=\boldsymbol{O}^{\prime}, \boldsymbol{\rho}_{\boldsymbol{O}^{\prime}, \mathfrak{B}}=\rho_{\boldsymbol{O}, \mathfrak{B}} .
$$

It follows from the second equation (22) that $\rho_{\boldsymbol{O}, \mathfrak{B}} \circ \rho_{\boldsymbol{O}, \mathfrak{B}}$ is the identity on every $\mathfrak{B}^{\prime} \mid \mathfrak{B}$, hence on the whole of $\mathfrak{W}$,

$$
\rho_{\boldsymbol{O}, \mathfrak{B}} \circ \rho_{\boldsymbol{O}, \mathfrak{z}}=\varepsilon \text {. }
$$

We denote by $\Re_{\boldsymbol{O}, \mathfrak{g}}$ the set of all particles $\boldsymbol{O}^{\prime} \| \boldsymbol{O}$ with the property (22) and by $\left|\Re_{\boldsymbol{O}, \mathfrak{P}}\right|$ the union of all sets of events $\left|\boldsymbol{O}^{\prime}\right|, \boldsymbol{O}^{\prime} \in \Re_{\boldsymbol{o}, \mathfrak{g}}$. Thus with each reflection $\rho_{\boldsymbol{O}, \mathfrak{g}}$ we associate a "plane of reflection" $\mathfrak{\Re}_{\boldsymbol{o}, \mathfrak{g}}$ consisting of all particles $\boldsymbol{O}^{\prime} \| \boldsymbol{O}$ invariant under $\rho_{\boldsymbol{o}, \mathfrak{3}}$. Because of Al2, $\Re_{\boldsymbol{O}, \mathfrak{B}}$ consists of at least two particles.

Theorem 18. Let $\mathfrak{B}=\mathfrak{B}(\boldsymbol{O}, \boldsymbol{P}) \in \mathscr{V}(\boldsymbol{O}), \lambda>0$. Then

$$
\sigma_{\boldsymbol{O}, \boldsymbol{P}, 2 \lambda} \circ \rho_{\boldsymbol{O}, \mathfrak{z}}=\rho_{\boldsymbol{O}_{1}, \mathfrak{z}} \text { where } \boldsymbol{O}_{1}=\sigma_{\boldsymbol{O}, \boldsymbol{p}, \lambda} \circ \boldsymbol{O} \text {. }
$$


By direct calculation one can verify that the automorphism on the left hand side of (24), has the properties required by A14, with $O_{1}$ replacing $\boldsymbol{O}$. Because of the uniqueness postulated in A14, it must be equal to $\rho_{\boldsymbol{o}_{1}, \mathfrak{g}}$.

The theorem shows that the space translation $\sigma_{\boldsymbol{o}, \boldsymbol{P}, \lambda}$ transfers $\Re_{\boldsymbol{o}, \boldsymbol{z}}$ into another plane of reflection $\Re_{\boldsymbol{O}_{1, \mathfrak{y}}}$ where $\boldsymbol{O}_{\mathbf{1}}|| \boldsymbol{O}$ and $\left|\Re_{\boldsymbol{O}, \mathfrak{y}}\right| \cap\left|\Re_{\boldsymbol{O}_{1, \mathfrak{y}}}\right|=\emptyset$. We say that $\Re_{\boldsymbol{o}, \boldsymbol{y}}$ and $\Re_{\boldsymbol{O}_{1, \boldsymbol{B}}}$ are parallel planes of reflection and write $\Re_{\boldsymbol{o}, \mathfrak{g}} \mid \Re_{\boldsymbol{o}_{1}, \mathfrak{B}} ;$ their distance is defined

$$
d\left(\Re_{\boldsymbol{o}, \mathfrak{B}}, \Re_{\boldsymbol{O}_{1}, \mathfrak{g}}\right)=|\lambda| \text { where } \boldsymbol{O}_{\mathbf{1}}=\sigma_{\boldsymbol{o}, \boldsymbol{p}, 2} \circ \boldsymbol{O}, \mathfrak{B}=\mathfrak{B}(\boldsymbol{O}, \boldsymbol{P}) .
$$

Clearly parallelity of planes of reflection is an equivalence relation and $\mathfrak{W}$ is the union of all $\Re_{\boldsymbol{o}_{1, \mathfrak{9}}}$ which are parallel to a fixed $\Re_{\boldsymbol{o}, \mathfrak{g}}$.

THEOREM 19. If $N \in \mathcal{N}^{*}$ and $|\boldsymbol{N}|$ has two distinct events in common with the hyperplane of reflection $\left|\Re_{\boldsymbol{o , g}}\right|$ then $|\boldsymbol{N}| \subset\left|\Re_{\boldsymbol{o}, \mathfrak{g}}\right|$.

For $N_{1}=\rho_{o, \mathfrak{B}} \circ N \in \mathscr{N}^{*}$ since $\rho_{o, \mathfrak{B}} \in \mathfrak{A}$, and $N_{1}\left(t_{i}\right)=N\left(t_{i}\right), i=1,2$ for some $t_{1} \neq t_{2}$, hence $N_{1}=N$, all events of $N$ are left unchanged by $\rho_{o, \mathfrak{v}}$.

Now given $\boldsymbol{O}^{\prime} \in \mathcal{O}^{*}, q \in \mathfrak{B}, q \notin\left|\boldsymbol{O}^{\prime}\right|$, let $\left\{\boldsymbol{P}_{\mathbf{1}}\right\}$ be the unique l.s. from $\boldsymbol{O}^{\prime}$ to $q$ and let $p=\left|\boldsymbol{O}^{\prime}\right| \cap\left|\boldsymbol{P}_{\mathbf{1}}\right|$. Then there is a uniquely determined local time $t$ on $\left\{\boldsymbol{O}^{\prime}\right\}$ and a particle $\boldsymbol{O}(t)=\boldsymbol{O}^{\prime}\left(t^{\prime}\right)$ such that $p=\boldsymbol{O}(0), q=\boldsymbol{P}^{\prime}(\mathbf{1})$ where $\left\{\boldsymbol{P}^{\prime}\right\}=\left\{\boldsymbol{P}_{\mathbf{1}}\right\}$ and $\boldsymbol{P}^{\prime}$ is synchronized with $\boldsymbol{O}$.

With these specifications let $\mathfrak{B}^{\prime}=\mathfrak{B}\left(\boldsymbol{O}, \boldsymbol{P}^{\prime}\right)$ and $\mathfrak{R}_{\boldsymbol{o}, \mathfrak{y}^{\prime}}$ the corresponding plane of reflection. Given any $\boldsymbol{O}_{1} \in \Re_{\boldsymbol{o}, \mathfrak{y}^{\prime}} \boldsymbol{O}_{\mathbf{1}} \neq \boldsymbol{O}$, let $\boldsymbol{P}$ be the photon from $p$ to $\boldsymbol{O}_{1}$, synchronized with $\boldsymbol{O}$. Since $\boldsymbol{P}$ has two distinct events in common with $\left|\Re_{\boldsymbol{o}, \mathfrak{g}^{\prime}}\right|,|\boldsymbol{P}| \subset\left|\Re_{\boldsymbol{O}, \mathfrak{g}^{\prime}}\right|$ by Theorem 19 . We call the configuration $[q, \boldsymbol{O}, \boldsymbol{P}]$ with the above specifications a cross of reference.

\section{A15 (AXIOM OF ISOTROPY)}

Given two crosses of reference $\left[q_{i}, \boldsymbol{O}_{i}, \boldsymbol{P}_{i}\right], i=1,2$ there are precisely two automorphisms $\phi_{i}, i=1,2$ such that

$$
\phi_{i}\left(q_{1}\right)=q_{2}, \phi_{i} \circ \boldsymbol{O}_{1}=\boldsymbol{O}_{2}, \phi_{i} \circ \boldsymbol{P}_{1}=\boldsymbol{P}_{2} .
$$

Moreover

$$
\phi_{1} \circ \phi_{2}^{-1}=\phi_{2} \circ \phi_{1}^{-1}=\rho_{o_{2}, \mathfrak{B}}
$$

for some $\mathfrak{B} \in \mathscr{V}\left(\boldsymbol{O}_{\mathbf{2}}\right), \quad|\boldsymbol{V}| \subset \Re_{\boldsymbol{o}_{2}, \mathfrak{y}^{\prime}} \mid$ where $\mathfrak{B}^{\prime} \in \mathscr{V}\left(\boldsymbol{O}_{2}\right)$ is the pseudoplane determined by $q_{2}$ and $\boldsymbol{O}_{2}$.

This is the last of our axioms; it expresses the complete isotropy of observers and light signals with respect to space positions and directions. The fact that there are exactly two automorphisms of the required kind which only differ by a reflection, ensures that $\mathfrak{B}$ is exactly four dimensional.

It follows from (26) and (27) that

$$
\rho_{\boldsymbol{O}_{2}, \mathfrak{y}}\left(q_{2}\right)=q_{2}, \rho_{\boldsymbol{O}_{2}, \mathfrak{3}} \circ \boldsymbol{P}_{2}=\boldsymbol{P}_{2}
$$


hence $q_{\mathbf{2}} \in\left|\Re_{\boldsymbol{o}_{\mathbf{g}}, \mathfrak{v}}\right|,\left|\boldsymbol{P}_{\mathbf{2}}\right| \subset\left|\Re_{\boldsymbol{o}_{3}, \mathfrak{g}}\right|$. If in particular we take $q_{1}=q_{2}, \boldsymbol{O}_{\mathbf{1}}=\boldsymbol{O}_{2}$, $\boldsymbol{P}_{\mathbf{1}}=\boldsymbol{P}_{\mathbf{2}}$ in A15, then one of the $\phi_{i}$ is the identity automorphism. By setting $\phi_{1}=\varepsilon, \phi_{2}=\rho$ we obtain

THEOREM 20. Given a cross of reference $[q, \boldsymbol{O}, \boldsymbol{P}]$ there is a unique reflection $\rho=\rho_{\boldsymbol{O}, \mathfrak{s}_{0}}$ with the following properties:

(i) $q \in\left|\Re_{\boldsymbol{o}, \mathfrak{\vartheta}_{0}}\right|,|\boldsymbol{P}| \subset\left|\Re_{\boldsymbol{o}, \mathfrak{\vartheta}_{0}}\right|$.

(ii) $\left|\mathfrak{B}_{\boldsymbol{o}}\right| \subset\left|\Re_{\boldsymbol{o}, \mathfrak{g}^{\prime}}\right|$ where $q \in\left|\mathfrak{B}^{\prime}\right|$.

We denote this reflection by $\rho_{0}(q, \boldsymbol{O}, \boldsymbol{P})$; the pseudoplane $\mathfrak{B}_{\mathbf{0}}$ is said to be orthogonal to the cross $[q, \boldsymbol{O}, \boldsymbol{P}]$. Thus with every cross of reference $[q, \boldsymbol{O}, \boldsymbol{P}]$ we associate uniquely three reflections:

$$
\begin{aligned}
& \rho_{0}=\rho_{0}(q, \boldsymbol{O}, \boldsymbol{P})=\rho_{\boldsymbol{o}, \mathfrak{y}_{0}}, \\
& \rho_{\mathbf{1}}=\rho_{\mathbf{1}}(q, \boldsymbol{O}, \boldsymbol{P})=\rho_{\boldsymbol{O}, \mathfrak{z}_{\mathbf{1}}} \text { where } \mathfrak{V}_{1}=\mathfrak{B}(\boldsymbol{O}, \boldsymbol{P}) \\
& \rho_{2}=\rho_{2}(q, \boldsymbol{O}, \boldsymbol{P})=\rho_{\boldsymbol{O}, \mathfrak{y}_{2}} \text { where } \mathfrak{V}_{2}=\mathfrak{B}\left(\boldsymbol{O}, \boldsymbol{P}^{\prime}\right), q=\boldsymbol{P}^{\prime}(1) \text {. }
\end{aligned}
$$

Let $\mathfrak{B}_{\mathbf{0}}=\mathfrak{B}\left(\boldsymbol{O}, \boldsymbol{P}^{\prime \prime}\right)$ and set $q_{0}=\boldsymbol{P}^{\prime \prime}(1), q_{1}=\boldsymbol{P}(1)$. Then $\zeta_{0} \circ \rho_{0} \circ \rho_{2}$ is an automorphism which leaves $q$ and $q_{0}$ invariant and carries $q_{1}$ into its reflection with respect to $\boldsymbol{O}$. But $\left[q, \boldsymbol{O}, \boldsymbol{P}^{\prime \prime}\right]$ is a cross of reference and therefore by Theorem 20 the unique automorphism $\neq \varepsilon$ which leaves the cross invariant is the reflection $\rho_{0}\left(q, \boldsymbol{O}, \boldsymbol{P}^{\prime \prime}\right)$. Since it carries $q_{1}$ into its reflection with respect to $\boldsymbol{O}$, it must be identical with $\rho_{\boldsymbol{O}, \mathfrak{B}_{1}}=\rho_{\mathbf{1}}$. The same is true of $\zeta_{0} \circ \rho_{2} \circ \rho_{0}$ and in fact of any permutation of these three automorphisms. Hence $\zeta_{\boldsymbol{o}}, \rho_{0}, \rho_{1}, \rho_{2}$ mutually commute and $\rho_{0} \circ \rho_{1} \circ \rho_{2}=\zeta_{\boldsymbol{o}}$. Thus we have

THEOREM 21. With every cross of reference $[q, \boldsymbol{O}, \boldsymbol{P}]$ there are uniquely associated three reflections $\rho_{i}=\rho_{\boldsymbol{o}}, \mathfrak{x}_{i}, i=0,1,2$, satisfying

$$
\begin{aligned}
& \rho_{i} \circ \rho_{j}=\rho_{j} \circ \rho_{i}, \\
& \rho_{0} \circ \rho_{1} \circ \rho_{\mathbf{2}}=\zeta_{\boldsymbol{O}}
\end{aligned}
$$

and having the following property:

If $\mathfrak{B}_{\boldsymbol{i}}=\mathfrak{B}\left(\boldsymbol{O}, \boldsymbol{P}_{\boldsymbol{i}}\right)$ where $\boldsymbol{P}_{\boldsymbol{i}}(0)=\boldsymbol{O}(0)$, and $q_{i}=\boldsymbol{P}_{\boldsymbol{i}}(\mathbf{1})$, then each $\left[q_{i}, \boldsymbol{O}, \boldsymbol{P}_{i}\right], i \neq j, i, j=0,1,2$ is a cross of reference with $\mathfrak{B}_{k}, k \neq i, k \neq j$ orthogonal to $\left[q_{i}, \boldsymbol{O}, \boldsymbol{P}_{j}\right]$ and $\left[q_{1}, \boldsymbol{O}, \boldsymbol{P}_{2}\right]=[q, \boldsymbol{O}, \boldsymbol{P}]$.

\section{Properties of 3-space}

We have seen (Theorem 9) that $\boldsymbol{O} \| \boldsymbol{O}^{\prime}$ is an equivalence relation; we denote by $\Re(\boldsymbol{O})$ the equivalence class represented by $\boldsymbol{O} . \Re(\boldsymbol{O})$ is the space of simultaneity relative to $\boldsymbol{O}$; thus $\boldsymbol{O}^{\prime}$ is a point of $\Re(\boldsymbol{O}), \boldsymbol{O}^{\prime} \in \Re(\boldsymbol{O})$, iff $\boldsymbol{O}^{\prime} \in \mathcal{O}^{*}$ and $\boldsymbol{O}^{\prime} \| \boldsymbol{O}$. Clearly $\mathfrak{R}\left(\boldsymbol{O}^{\prime}\right)=\mathfrak{R}(\boldsymbol{O})$ for every $\boldsymbol{O}^{\prime} \| \boldsymbol{O}$. From Theorem 
7 it follows that through every $q \in \mathfrak{W}$ there passes exactly one $\boldsymbol{O}^{\prime} \in \Re(\boldsymbol{O})$ so that $\mathfrak{W}$ is the set union of all $\left|\boldsymbol{O}^{\prime}\right|, \boldsymbol{O}^{\prime} \in \mathfrak{R}(\boldsymbol{O})$. We shall now study the geometry of $\Re(\boldsymbol{O})$.

A straight line (or simply a line) in $\Re(O)$ consists of the set of points $\boldsymbol{O}^{\prime}$ which intersect (in $\mathfrak{B}$ ) a given photon $\boldsymbol{P}$. All photons in $\mathfrak{B}\left(\boldsymbol{O}^{\prime}, \boldsymbol{P}\right)$ define the same line, by Axiom A7 (and its corollaries Theorems 4 and 5 ), so that there is a one to one correspondence between lines in $\Re(O)$ and pairs of conjugate pseudoplanes $\left[\mathfrak{B}, \mathfrak{B}^{*}\right]$, both in $\mathfrak{B}\left(\boldsymbol{O}^{\prime}\right)$ where $\boldsymbol{O}^{\prime} \in \Re(\boldsymbol{O})$. We denote by $\overline{\mathscr{L}}(\boldsymbol{O})$ the set of lines in $\Re(\boldsymbol{O})$, and by $\mathscr{L}(\boldsymbol{O})$ the set of directed lines, i.e. lines together with a sense of ordering of the points on the lines, induced by the ordering on $\boldsymbol{P}$ in $\mathfrak{B}\left(\boldsymbol{O}^{\prime}, \boldsymbol{P}\right)$. Hence there is a one to one correspondence between directed lines in $\Re(\boldsymbol{O})$ and pseudoplanes $\mathfrak{B} \in \mathscr{V}\left(\boldsymbol{O}^{\prime}\right)$, $\boldsymbol{O}^{\prime} \in \mathfrak{R}(\boldsymbol{O})$. Identifying these two we write $\mathfrak{B} \in \mathscr{L}(\boldsymbol{O})$. As before, $|\mathfrak{B}|$ denotes the set of events contained in the corresponding $\mathfrak{B}\left(\boldsymbol{O}^{\prime}, \boldsymbol{P}\right)$.

The ordering of points on a directed line is isomorphic to the ordering on the real line via the parameter $\delta$ in (10). Hence the usual properties of betweenness of points on a line hold, also the Archimedean axiom. Clearly this ordering is independent of the standard local time on $O$, and is reversed for the conjugate line $\mathfrak{B}^{*}$ in which the roles of $\boldsymbol{P}$ and $\boldsymbol{P}^{*}$ are interchanged.

Distance of the points $\boldsymbol{O}^{\prime}, \boldsymbol{O}^{\prime \prime} \in \mathfrak{R}(\boldsymbol{O})$ is also defined through the parameter $\delta$, namely by $d\left(\boldsymbol{O}^{\prime}, \boldsymbol{O}^{\prime \prime}\right)=|\delta|$, in accordance with our earlier notation. The unit in which the distance is expressed depends of course on the local time on $\boldsymbol{O}$.

THEOREM 22. Through any two distinct points $\boldsymbol{O}^{\prime}, \boldsymbol{O}^{\prime \prime} \in \Re(O)$ there goes exactly one line.

The theorem follows from the fact that two distinct parallel observers uniquely determine the pseudoplane $|\mathfrak{B}|$ in which they are contained. For if $q \in\left|\boldsymbol{O}^{\prime \prime}\right|$ and $\{\boldsymbol{P}\}$ is a l.s. through $q$ which meets $\boldsymbol{O}^{\prime}$ then by the definition of parallelity $\boldsymbol{O}^{\prime \prime}$ must lie in $\left|\mathfrak{B}\left(\boldsymbol{O}^{\prime}, \boldsymbol{P}\right)\right|$ and by Theorems 4 and 5 the same $|\mathfrak{B}|$ is obtained from every $q \in\left|\boldsymbol{O}^{\prime \prime}\right|$.

COROLlary. Two distinct lines meet in at most one point.

THEOREM 23. Given distinct points $\boldsymbol{O}_{1}, \boldsymbol{O}_{2} \in \Re(\boldsymbol{O})$ there exists a point $\mathrm{O}_{3}$ not on the line through $\mathrm{O}_{1}, \mathrm{O}_{2}$. Furthermore

$$
d\left(\boldsymbol{O}_{2}, \boldsymbol{O}_{3}\right)<d\left(\boldsymbol{O}_{1}, \boldsymbol{O}_{2}\right)+d\left(\boldsymbol{O}_{1}, \boldsymbol{O}_{3}\right) .
$$

Proof. By Al2 there is an event $q$ not in the pseudoplane $|\mathfrak{B}|$ through $\boldsymbol{O}_{1}$ and $\boldsymbol{O}_{2}$, and by Theorem 7 there is an $\boldsymbol{O}_{3} \in \Re(\boldsymbol{O})$ through $q$. This proves our first statement.

By A4 there is a l.s. $\{P\}$ from $O_{1}$ to $q$ (hence not in $|\mathfrak{B}|$ ). Let $p_{1}=|\boldsymbol{P}| \cap\left|\boldsymbol{O}_{1}\right|,\left\{\boldsymbol{P}_{1}\right\}$ a l.s. from $\boldsymbol{O}_{2}$ to $\boldsymbol{P}_{1}, p_{2}=\left|\boldsymbol{P}_{1}\right| \cap\left|\boldsymbol{O}_{2}\right|, \boldsymbol{O}^{\prime}$ a particle 
from $p_{2}$ to $q$ (which exists by A5), $\left\{\boldsymbol{P}_{2}\right\}$ a l.s. from $p_{2}$ to $\boldsymbol{O}_{3}, q^{\prime}=\left|\boldsymbol{O}_{3}\right| \cap\left|\boldsymbol{P}_{2}\right|$. We have $p_{2}<p_{1}, p_{1}<q, p_{2}<q, p_{2}<q^{\prime}, q^{\prime}<q$ by Theorem 1 . Furthermore we have the equations

$$
\begin{aligned}
t\left(\boldsymbol{O}, p_{1}\right) & =t\left(\boldsymbol{O}, p_{2}\right)+d\left(\boldsymbol{O}_{1}, \boldsymbol{O}_{2}\right), \\
t\left(\boldsymbol{O}, p_{1}\right)+d\left(\boldsymbol{O}_{1}, \boldsymbol{O}_{3}\right) & =t(\boldsymbol{O}, q)>t\left(\boldsymbol{O}, q^{\prime}\right)=t\left(\boldsymbol{O}, p_{2}\right)+d\left(\boldsymbol{O}_{2}, \boldsymbol{O}_{3}\right),
\end{aligned}
$$

which give the triangle inequality (30). Thus $\Re(O)$ is endowed with a metric topology, derived from the distance function $d\left(\boldsymbol{O}_{1}, \boldsymbol{O}_{2}\right)$.

Two directed lines $\mathfrak{B}_{1}, \mathfrak{B}_{2}$ are called parallel and denoted $\mathfrak{B}_{1} \mid \mathfrak{B}_{2}$ iff the corresponding pseudoplanes in $\mathfrak{B}$ are parallel, antiparallel iff $\mathfrak{B}_{1} \mid \mathfrak{B}_{2}^{*}$. Theorem 12 shows that $\mathfrak{B}_{1} \mid \mathfrak{B}_{2}$ is an equivalence relation. Theorem 13 gives

THEOREM 24. Given $\mathfrak{B} \in \mathscr{L}(\boldsymbol{O})$ and $\boldsymbol{O}^{\prime} \in \mathfrak{R}(\boldsymbol{O})$, there is exactly one directed line $\mathfrak{B}^{\prime} \in \mathscr{L}(\boldsymbol{O})$ such that $\boldsymbol{O}^{\prime} \in \mathfrak{B}^{\prime}$ and $\mathfrak{B}^{\prime} \mid \mathfrak{B}$.

This of course is the well known Euclidean parallel postulate which appears here as a theorem.

A one to one mapping $\psi$ of $\Re(O)$ onto $\Re(O)$ is called a similarity iff it carries lines into lines and preserves the ratio of distances. Hence

and

$$
\mathfrak{B} \in \mathscr{L}(\boldsymbol{O}) \Rightarrow \psi(\mathfrak{B}) \in \mathscr{L}(\boldsymbol{O})
$$

$$
d\left(\psi\left(\boldsymbol{O}_{1}\right), \psi\left(\boldsymbol{O}_{2}\right)\right) / d\left(\boldsymbol{O}_{1}, \boldsymbol{O}_{2}\right)=\gamma>0
$$

for all $\boldsymbol{O}_{1}, \boldsymbol{O}_{2} \in \Re(O), \boldsymbol{O}_{1} \neq \boldsymbol{O}_{2}$, where $\gamma$ is independent of $\boldsymbol{O}_{\mathbf{1}}$ and $\boldsymbol{O}_{\mathbf{2}}$. If in particular $\gamma=1$, i.e. $\psi$ preserves distances, then it is called a congruence. Clearly a similarity is a homeomorphism $\mathfrak{R}(\boldsymbol{O}) \rightarrow \mathfrak{R}(\boldsymbol{O})$ under the metric topology.

Suppose now that $\phi$ is an automorphism of $\mathfrak{W}$, admitting $\boldsymbol{O}$. By Theorem 16 it admits $\boldsymbol{O}_{1}$ for all $\boldsymbol{O}_{1} \in \Re(O)$ and maps $\boldsymbol{O}_{1}$ into a particle $\phi \circ \boldsymbol{O}_{1}$ which by Theorem 15 is homographic to an $\boldsymbol{O}_{1}^{\prime} \in \Re(\boldsymbol{O})$. Thus it defines uniquely a one to one mapping $\phi^{*}$ of $\Re(\boldsymbol{O})$ onto $\Re(\boldsymbol{O})$, through $\phi^{*} \circ O_{1}=O_{1}^{\prime}$. Since by Theorem $15 \phi$ maps pseudoplanes into pseudoplanes, $\phi^{*}$ maps lines of $\Re(\boldsymbol{O})$ onto lines. Furthermore it follows from the corollary of Theorem 16 that $d\left(\phi^{*}\left(\boldsymbol{O}_{1}\right), \phi^{*}\left(\boldsymbol{O}_{2}\right)\right)=\gamma d\left(\boldsymbol{O}_{1}, \boldsymbol{O}_{2}\right)$ for all $\boldsymbol{O}_{1}, \boldsymbol{O}_{2} \in \Re(\boldsymbol{O})$ and a fixed positive constant $\gamma$. Hence we obtain

THEOREM 25. If $\phi$ is an automorphism of $\mathfrak{W}$ admitting $\boldsymbol{O}$, and $\phi^{*}$ is the associated mapping $\Re(O) \rightarrow \Re(O)$ then $\phi^{*}$ is a similarity.

Conversely, given a similarity $\psi$ of $\Re(O)$, we can extend it to an automorphism $\phi$ of $\mathfrak{B}$ by the rule: If $q=\boldsymbol{O}_{\mathbf{1}}(t), \boldsymbol{O}_{\mathbf{1}} \| \boldsymbol{O}$, then $\phi(q)=\psi \circ \boldsymbol{O}_{\mathbf{1}}(\gamma t)$. It is easy to verify (by reverting the proof of Theorem 16) that $\phi$ is in fact an automorphism and that $\psi=\phi^{*}$.

The congruence $\sigma^{*}$ corresponding to a space translation $\sigma_{\boldsymbol{O}^{\prime} ; \boldsymbol{P}, \lambda}, \boldsymbol{O}^{\prime} \in \Re(\boldsymbol{O})$ 
is a vector translation characterized by the direction $\mathfrak{B}\left(\boldsymbol{O}^{\prime}, \boldsymbol{P}\right)$ and magnitude $\lambda$. Similarly to $\zeta_{\boldsymbol{o}}, \boldsymbol{O}^{\prime} \in \Re(\boldsymbol{O})$ and $\rho_{\boldsymbol{o}^{\prime}, \mathfrak{g}}, \mathfrak{B} \in \mathscr{V}(\boldsymbol{O})^{\prime}$, there corresponds a central reflection $\zeta_{\boldsymbol{o}}^{*}$, and space reflection $\rho_{\boldsymbol{O}^{\prime}, \mathfrak{B}}^{*}$ of $\Re(\boldsymbol{O})$. The first one is a congruence which leaves $O^{\prime}$ invariant and carries $O_{1} \in \Re(O), O_{1} \neq O^{\prime}$ into $\boldsymbol{O}_{2}=\zeta_{0}^{*},\left(\boldsymbol{O}_{1}\right) \neq \boldsymbol{O}_{1}$ with the property that $\boldsymbol{O}_{1}, \boldsymbol{O}^{\prime}, \boldsymbol{O}_{2}$ are collinear and $d\left(\boldsymbol{O}_{1}, \boldsymbol{O}^{\prime}\right)=d\left(\boldsymbol{O}_{2}, \boldsymbol{O}^{\prime}\right)$. The second is a congruence which leaves all point of the plane of reflection $\Re_{\boldsymbol{o}^{\prime}, \mathfrak{B}}$ invariant. According to Axiom Al4 there is exactly one space reflection $\rho^{*}=\rho_{\boldsymbol{O}^{\prime}, \mathfrak{g}}^{*}$ which leaves $\boldsymbol{O}^{\prime}$ invariant and carries the directed line $\mathfrak{B}$ through $\boldsymbol{O}^{\prime}$ into its conjugate, reverting its direction.

If $\boldsymbol{O}_{1} \in \mathfrak{B}, \boldsymbol{O}_{1} \neq \boldsymbol{O}^{\prime}$, then $d\left(\boldsymbol{O}_{1}, \boldsymbol{O}^{\prime \prime}\right)>d\left(\boldsymbol{O}_{1}, \boldsymbol{O}^{\prime}\right)$ for every $\boldsymbol{O}^{\prime \prime} \neq \boldsymbol{O}^{\prime}$, $\boldsymbol{O}^{\prime \prime} \in \Re_{\boldsymbol{O}^{\prime}, \mathfrak{B}} ;$ for $\rho^{*}\left(\boldsymbol{O}^{\prime \prime}\right)=\boldsymbol{O}^{\prime \prime}, d\left(\boldsymbol{O}_{1}, \boldsymbol{O}^{\prime \prime}\right)=d\left(\boldsymbol{O}_{2}, \boldsymbol{O}^{\prime \prime}\right)$ where $\boldsymbol{O}_{2}=\rho^{*}\left(\boldsymbol{O}_{1}\right) \in \mathfrak{B}$ since $\rho^{*}$ is a congruence, and

$$
2 d\left(\boldsymbol{O}_{1}, \boldsymbol{O}^{\prime}\right)=d\left(\boldsymbol{O}_{1}, \boldsymbol{O}_{2}\right)<d\left(\boldsymbol{O}_{1}, \boldsymbol{O}^{\prime \prime}\right)+d\left(\boldsymbol{O}_{2}, \boldsymbol{O}^{\prime \prime}\right)=2 d\left(\boldsymbol{O}_{1}, \boldsymbol{O}^{\prime \prime}\right)
$$

by the triangle inequality. Thus $d\left(\boldsymbol{O}_{1}, \boldsymbol{O}^{\prime}\right)$ is the shortest distance of $\boldsymbol{O}_{1}$ from points of $\Re_{\boldsymbol{o}^{\prime}, \mathfrak{B}}$. We say that the line $\mathfrak{B}$ is perpendicular to the plane $\Re_{\boldsymbol{o}^{\prime}, \mathfrak{s}}$.

If $\boldsymbol{O}_{1}, \boldsymbol{O}_{2} \in \Re_{\boldsymbol{o}^{\prime}, \mathfrak{3}}, \boldsymbol{O}_{1} \neq \boldsymbol{O}_{2}$ then all points of the line $\mathfrak{B}^{\prime}$ joining $\boldsymbol{O}_{1}$ and $\boldsymbol{O}_{2}$ lie in $\Re_{\boldsymbol{O}^{\prime}, \mathfrak{g}}$. This follows from the fact that $\rho^{*}$ (being a congruence) carries $\mathfrak{B}^{\prime}$ into a line $\mathfrak{B}^{\prime \prime}$, but leaves two distinct points of $\mathfrak{B}^{\prime}$ invariant; hence $\mathfrak{B}^{\prime \prime}=\mathfrak{B}^{\prime}$ by Theorem 22 , and since distances are preserved, it must leave all points of $\mathfrak{B}^{\prime}$ invariant. If in particular $\boldsymbol{O}_{\mathbf{1}}=\boldsymbol{O}^{\prime}$ then $\mathfrak{B}$ is said to be perpendicular to $\mathfrak{B}^{\prime}$ at $\boldsymbol{O}^{\prime}$.

From Theorems 20 and 21 it follows that if $\mathfrak{B}$ is perpendicular to $\mathfrak{B}^{\prime}$ at $\boldsymbol{O}^{\prime}$ then also $\mathfrak{B}^{\prime}$ is perpendicular to $\mathfrak{B}$ at $\boldsymbol{O}^{\prime}$ and there is a unique (nondirected) line $\left[\mathfrak{B}^{\prime \prime}, \mathfrak{B}^{\prime *}\right]$ through $\boldsymbol{O}^{\prime}$ such that $\mathfrak{B}^{\prime \prime}$ is perpendicular to both $\mathfrak{Z}$ and $\mathfrak{B}^{\prime}$. From here the two-dimensionality of $\Re_{\boldsymbol{o}^{\prime}, \mathfrak{g}}$ and the three-dimensionality of $\Re(\boldsymbol{O})$ follows immediately.

It would lead us too far to develop the whole of the Euclidean system in a systematic fashion; we only mention the most essential points. From Theorem 18 it follows that all planes of symmetry $\Re_{\boldsymbol{o}_{1}, \mathfrak{z}}$ where $\boldsymbol{O}_{\mathbf{1}}$ is on $\mathfrak{B}$, can be obtained from $\Re_{\boldsymbol{o}^{\prime}, \mathfrak{Z}}$ by a vector displacement along $\mathfrak{B}$; and in particular, every $\boldsymbol{O}_{2} \in \Re(\boldsymbol{O})$ is contained in exactly one $\Re_{\boldsymbol{o}_{1}, \mathfrak{g}}$. It follows that from every $\boldsymbol{O}_{\mathbf{2}}$ not on $\mathfrak{B}$ one can drop a unique perpendicular $\mathfrak{B}^{\prime}$ upon $\mathfrak{B}$. The reflection of $\boldsymbol{O}_{2}$ with respect to $\mathfrak{B}$ is then obtained as the unique point $\boldsymbol{O}_{2}^{\prime}$ on $\mathfrak{B}^{\prime}, \boldsymbol{O}_{2}^{\prime} \neq \boldsymbol{O}_{2}$, such that $d\left(\boldsymbol{O}_{1}, \boldsymbol{O}_{2}\right)=d\left(\boldsymbol{O}_{1}, \boldsymbol{O}_{2}^{\prime}\right)$; the mapping $\eta_{\mathfrak{B}}^{*}\left(\boldsymbol{O}_{2}\right)=\boldsymbol{O}_{2}^{\prime}$ is called a half-turn about $\mathfrak{B}$. It can be identified as

$$
\eta_{\mathfrak{B}}^{*}=\zeta_{\boldsymbol{o}^{\prime}}^{*} \circ \rho_{\boldsymbol{o}^{\prime}, \mathfrak{B}}^{*}=\rho_{\boldsymbol{o}^{\prime}, \mathfrak{B}}^{*} \circ \zeta_{\boldsymbol{o}^{\prime}}^{*}
$$

where $\boldsymbol{O}^{\prime}$ is any point on $\mathfrak{B}$. Hence a half-turn is a congruence which carries lines in $\Re_{\boldsymbol{o}^{\prime}, \mathfrak{B}}$ into their (parallel) reflection in $\Re_{\boldsymbol{o}^{\prime}, \mathfrak{B}}$ with respect to $\boldsymbol{O}^{\prime}$. 
From here all the laws of alternate, opposite and complementary angles can be derived, and hence the usual properties of similar triangles, including the angle-sum. As a result we obtain Pythagoras and the whole of coordinate geometry. A detailed discussion is omitted.

\section{Appendix}

The following remarkable theorem is due to M. L. Urquhart; he gave an account of it in a symposium on relativity at the 1964 meeting of the Australian Mathematical Society in Adelaide.

TheOREM OF URquhart. Let $\boldsymbol{O} \in \mathcal{O}, \gamma \in|\boldsymbol{O}|,\{\boldsymbol{P}\}$ a l.s. through $q$, $q_{1} \in|\boldsymbol{P}|, q_{\mathbf{1}}>q, \quad\left\{\boldsymbol{P}_{\mathbf{1}}\right\}$ a l.s. through $q_{1}$ but not lying in $\mathfrak{B}_{\mathbf{1}}=\mathfrak{B}(\boldsymbol{O}, \boldsymbol{P})$, $q_{2} \in\left|\boldsymbol{P}_{1}\right|, q_{2}>q_{1},\left\{\boldsymbol{P}_{2}\right\}$ a l.s. from $q_{2}$ to $\boldsymbol{O}$.

Let $\{\boldsymbol{O}\}$ be an observer through $q_{2}$ in $\mathfrak{B}_{2}=\mathfrak{B}\left(\boldsymbol{O}, \boldsymbol{P}_{2}\right),\left\{\boldsymbol{P}_{2}^{*}\right\}$ the l.s. from $q$ to $\boldsymbol{O}^{\prime}, p_{\mathbf{2}}=\left|\boldsymbol{O}^{\prime}\right| \cap\left|\boldsymbol{P}_{2}^{*}\right|,\left\{\boldsymbol{P}_{1}^{*}\right\}$ the l.s. through $p_{\mathbf{2}}$ intersecting $\boldsymbol{P}_{\mathbf{1}}$. Then

(i) $\boldsymbol{P}_{1}^{*}$ intersects $\mathfrak{B}_{1}$.

(ii) If $p_{1}=\left|\boldsymbol{P}_{1}^{*}\right| \cap\left|\mathfrak{B}_{1}\right|$ and $\left\{\boldsymbol{P}^{*}\right\}$ is the l.s. from $p_{\mathbf{1}}$ to $\boldsymbol{O}$ then

$$
\left|\boldsymbol{P}^{*}\right| \cap|\boldsymbol{O}|=\left|\boldsymbol{P}_{2}^{*}\right| \cap|\boldsymbol{O}| \text {. }
$$

The essential part of Urquhart's theorem is in the second statement; the first statement is merely a kinematic formulation of Euclid's main incidence axiom of the plane. The significance of the theorem is that it can be formulated (though not proved) from the existence and incidence axioms alone ( $\mathrm{Al}-\mathrm{A} 6$ and $\mathrm{A} 12)$ without assuming standard time, synchronization and symmetry. Urquhart himself took the view that it should be taken as one of the kinematic axioms. ${ }^{2}$ At any rate it seems likely that any part of the Euclidean system which cannot be derived from axioms $\mathrm{A} 1-\mathrm{A} 12$ and this theorem, cannot be obtained from purely kinematical observations.

In Euclidean terms the theorem states the following:

URQUHART'S THEOREM, GEOMETRICAL FORM. If in the configuration shown (Fig. 1)

$$
A E+E B=A F+F B
$$

then

$$
A C+C F+F A=A E+E D+D A .
$$

The following is Urquhart's proof, as reconstructed and simplified by Professor B. C. Rennie. The proof rests on the following Lemma:

2 A trivial form of the theorem (assuming $O^{\prime}$ to be parallel to $O$ ) appears as a postulate in Robb's system [3, p. 167]. 
Given a circle and given two points $A$ and $C$ outside the circle, the line $A C$ will be a tangent if the distance $A C$ equals the difference between (or the sum of) the length of a tangent from $A$ and the length of a tangent from $C$.

To prove the Lemma, draw a tangent $A T$. On it take $S$ such that $A S=A C$. By assumption $S T=$ the length of tangent from $C$. It follows therefore that if $O$ is the centre of the circle, $S O=C O$, the triangles $O A S$ and $O A C$ are congruent and angle $O A S=$ angle $O A C$. Hence $A C$ is a tangent.

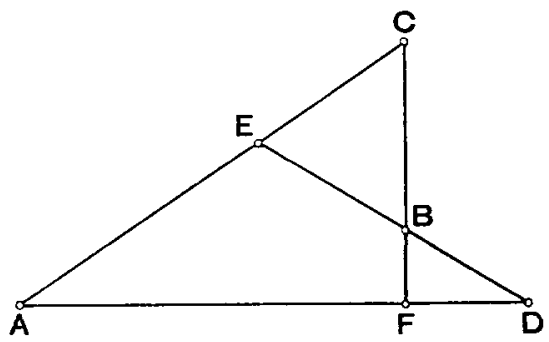

Fig. 1

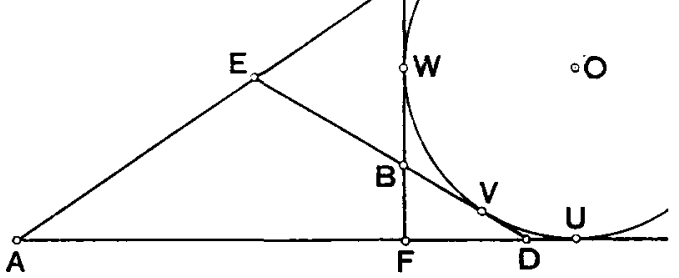

Fig. 2

Now suppose that (A1) holds; then the four sides of the complete quadrilateral (marked $A, B, C, D, E, F$ in Fig. 2) all touch a circle. For draw a circle touching the three sides $A F D$ (at $U$ ), $E B D$ (at $V$ ) and $F B C$ (at $W$ ). Then $A U-E V=A E+F W-(E B+B W)=A F+F B-E B=A E$ hence by the Lemma $A E$ is tangent to the circle. Consequently

$$
A C+C B=(A U-C W)+C B=A U+B V=A D+D V+B V=A D+D B
$$

and (A2) follows.

\section{References}

[1] W. Noll, 'Euclidean geometry and Minkowskian chronometry', Amer. Math. Monthly 71 (1964), 129-144.

[2] H. Weyl, Space-Time-Matter (London, 1922).

[3] A. A. Robb, Geometry of time and space (Cambridge, 1936).

Department of Mathematics

University of New South Wales

Kensington, N.S.W. 